\title{
Passive Flow Control on a Ground-Effect Diffuser Using an Inverted Wing
}

In this experimental and computational study a novel application of aerodynamic principles in altering the pressure recovery behavior of an automotive-type ground-effect diffuser was investigated as a means of enhancing downforce. The proposed way of augmenting diffuser downforce production is to induce in its pressure recovery action a second pressure drop and an accompanying pressure rise region close to the diffuser exit. To investigate this concept with a diffuser-equipped bluff body, an inverted wing was situated within the diffuser flow channel, close to the diffuser exit. The wing's suction surface acts as a passive flow control device by increasing streamwise flow velocity and reducing static pressure near the diffuser exit. Therefore, a second-stage pressure recovery develops along the diffuser's overall pressure recovery curve as the flow travels from the diffuser's low pressure, high velocity inlet to its high pressure, low velocity exit. Consequently, downforce production is increased with the use of the wing. Across the range of ride heights investigated, computational fluid dynamics simulations, validated against wind tunnel measurements, show an increase in downforce, with the increase reaching a high of about $12 \%$ relative to the baseline (without the wing). However, the increment in downforce occurred at relatively high ride heights but not once the diffuser started stalling at relatively low ride heights.

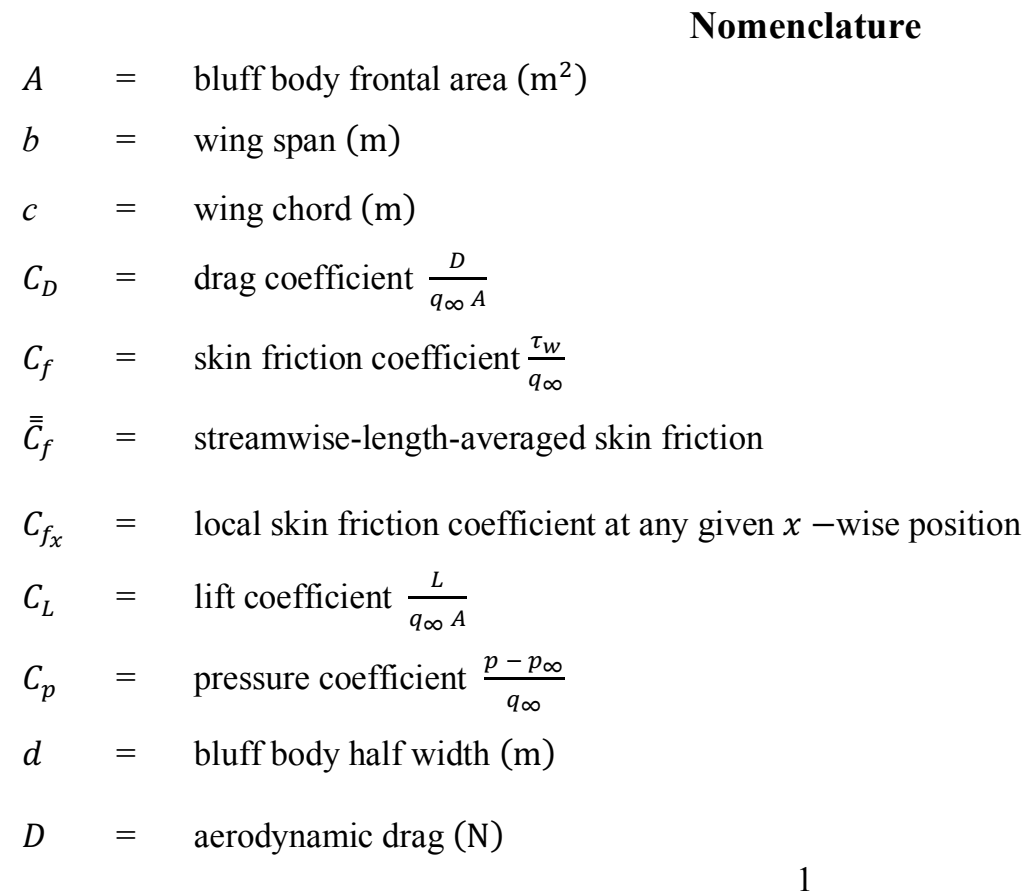


SAE Journal of Passenger Cars - Mechanical Systems

$h=$ bluff body ride height $(\mathrm{m})$

$H \quad=\quad$ bluff body height $(\mathrm{m})$

$L=$ aerodynamic downforce $(\mathrm{N})$

$L_{B} \quad=\quad$ bluff body length $(\mathrm{m})$

$L_{D} \quad=\quad$ diffuser length $(\mathrm{m})$

$p \quad=\quad$ static pressure $(\mathrm{Pa})$

$p_{\infty}=$ atmospheric pressure $(\mathrm{Pa})$

$q_{\infty}=$ freestream dynamic pressure $(\mathrm{Pa}), \frac{\rho U_{\infty}^{2}}{2}$

$R e=$ Reynolds number based on bluff body length $\frac{U_{\infty} L_{B}}{v}$

$u, \mathrm{v}, w=\quad$ velocity components in $x, y, z$ directions

$U=$ total velocity $\left(\mathrm{ms}^{-1}\right), \sqrt{u^{2}+\mathrm{v}^{2}+w^{2}}$

$U_{\theta}=$ total velocity parallel to the diffuser ramp surface $\left(\mathrm{ms}^{-1}\right), \sqrt{u_{\theta}^{2}+\mathrm{v}_{\theta}^{2}+w_{\theta}^{2}}$

$U_{\infty}=$ freestream velocity $\left(\mathrm{ms}^{-1}\right)$

$W \quad=\quad$ diffuser width $(\mathrm{m})$

$y^{+}=$dimensionless wall-normal distance

$x, y, z=$ Cartesian coordinates from origin (see Figure 2a): $x$ is positive downstream, $y$ is positive upwards, $z$ is positive to port side

\section{Greek Symbols}

$\alpha=$ angle of attack of the inverted wing relative to the chord line (degrees)

$\Delta_{t} \quad=\quad$ time-step (s)

$\Delta_{x} \quad=\quad$ smallest $x$-wise grid size $(\mathrm{m})$

$\theta \quad=\quad$ diffuser angle (degrees)

$\mu \quad=\quad$ dynamic viscosity $\left(\mathrm{kgm}^{-1} \mathrm{~s}^{-1}\right)$

$v=$ kinematic viscosity $\left(\mathrm{m}^{2} \mathrm{~s}^{-1}\right),\left(\frac{\mu}{\rho}\right)$

$\xi \quad=\quad$ local flow angle (degrees)

$\rho=$ air density $\left(\mathrm{kgm}^{-3}\right)$

$\tau_{w}=$ diffuser ramp shear stress $(P a), \mu\left(\frac{\partial U_{\theta}}{\partial y}\right)_{y=0}$

\section{Introduction}

$\mathrm{T}$ HE ground-effect diffuser is generally located at the aft section of a racing car's underbody. Unlike the planewalled diffuser with its equally diverging sides, the ground-effect diffuser is asymmetric in shape - typically 
SAE Journal of Passenger Cars - Mechanical Systems

consisting of a solitary diverging ramp surface. When in near proximity to the road surface, the diffuser becomes an increasing area duct, which provides a region in which the high-velocity/low-pressure underbody airflow entering the diffuser exits the diffuser as low velocity/high pressure airflow [1]. As a major automobile aerodynamic device, it enhances the aerodynamic performance of a Formula 1 racing car by generating the most downforce with the least drag penalty [2].

Downforce is generated by the ground-effect diffuser due to the suction effect created underneath the racing car. As the airflow with a high velocity travels underneath the smooth underbody-floor of the car, the diffuser area, which starts downstream of the floor, gradually decelerates the flow to low-velocity airflow at the exit of the diffuser [3]. As a result, the expanding area of the diffuser eases the low-pressure airflow at its inlet into higher pressure airflow at its exit. This creates a pressure recovery that begins from the suction peak (due to peak velocity) at the diffuser inlet to the higher pressure downstream of the inlet (due to reduced velocity) [4, 5]. It is this groundeffect phenomenon that generates downforce.

The flow features, downforce and drag behavior of the diffuser in ground effect have been described in 3-D ground-effect diffuser studies [4-12], often conducted on diffuser-equipped bluff bodies. George [6] discovered the formation of a longitudinal vortex pair along the lengthwise sides of the diffuser. When the effective angle of the diffuser was increased with an increase of the bluff body (nose-down) pitch, the vortices moved forward and strengthened due to the induced inflow, preventing the formation of a separation bubble on the diffuser ramp. George and Donis [7] found that sealing the sides of $10^{\circ}$ and $15^{\circ}$ diffusers to the ground with skirts obstructed inflow from the longitudinal-edge vortex pair, thus, stalling the diffuser flow. Senior et al. [8,9] discovered the existence of four distinct force regimes with equivalent diffuser flow behavior dependent on the gradual reduction of ride height from high to low. Investigations by Jowsey [10] indicated that the splitting of the diffuser flow channel with longitudinal fences enhanced downforce due to the generation of smaller longitudinal vortices, which in turn improve diffuser pumping and pressure recovery. CFD investigations by Puglisevich [11] using large eddy simulation (LES) correctly predicted the shape of the surface pressure distribution for the underbody and diffuser of the bluff body studied by Jowsey [10]. Experimental and CFD (using the Realizable $\kappa-\varepsilon$ turbulence model) studies of passenger cars and bluff bodies by Marklund [12] indicated that near-wake symmetry enabled the diffuser to achieve optimum pressure recovery.

The studies summarized above have mainly focused on 3-D diffusers with a plane surface and uninterrupted flow channel (no flow control). Thus, there is only a single pressure recovery from the diffuser inlet to its exit. For a single pressure recovery region, as illustrated in Fig. 1a, Cooper et al. [4, 5] formulated (in Eq. 1) the overall pressure-recovery coefficient $\left(\bar{C}_{p}\right)$ of the diffuser as: 


$$
\bar{C}_{p}=\frac{\left(C_{p 2}-C_{p 1}\right)}{\left(1-C_{p 1}\right)}
$$

However, as explained in [13], when a second pressure drop region is induced by flow control downstream of the initial pressure recovery originating at the diffuser inlet, the downforce generated by the diffuser is enhanced (Fig. 1b). This is because the average pressure of the two-stage diffuser is lower than that of the single-stage diffuser. Using Eq. (1), the overall pressure-recovery coefficient $\bar{C}_{p_{s}}$ for the diffuser with a two-stage pressure recovery can be written in Eq. (2) as:

$$
\bar{C}_{p_{s}}=\frac{\left(C_{p 3}-C_{p 1}\right)}{\left(1-C_{p 1}\right)}+\left[\frac{\left(C_{p 2}-C_{p 3}\right)}{\left(1-C_{p 3}\right)}\right] \frac{\left(1-C_{p 3}\right)}{\left(1-C_{p 1}\right)}
$$

As a means of inducing a second-stage pressure recovery, the velocity-pressure relationship close to the diffuser exit needs to be correspondingly altered. In this study, we induce the development of a static pressure drop and subsequent pressure recovery close to the diffuser exit with the novel use of the cambered suction surface of an inverted wing. As indicated in the studies on wall-bounded flows over convex surfaces [14-17], a pressure drop occurs with a subsequent reduction in wall friction, turbulence intensities and shear stresses. Therefore, the 2-D effects of an inverted wing (thickness, camber, and angle of attack) positioned across the diffuser flow channel, near to the diffuser exit and at an angle of attack equal to the diffuser ramp angle, can be employed to induce a static pressure drop. This is because, if the gap between the wing's pressure surface and the diffuser ramp surface is within $50 \%$ of the approximate boundary layer thickness then the wing's suction surface can increase the flow velocity close to the diffuser exit. The streamwise flow velocity increase correspondingly leads to a decrease in static pressure with a pressure increase towards the trailing edge of the wing. In this paper, the passive flow control method as described was employed on a bluff body equipped with a diffuser. Presented in this paper are wind tunnel results for force measurements, underbody surface pressures, and surface flow visualization, supported by CFD investigations validated with equivalent wind tunnel data. 
SAE Journal of Passenger Cars - Mechanical Systems
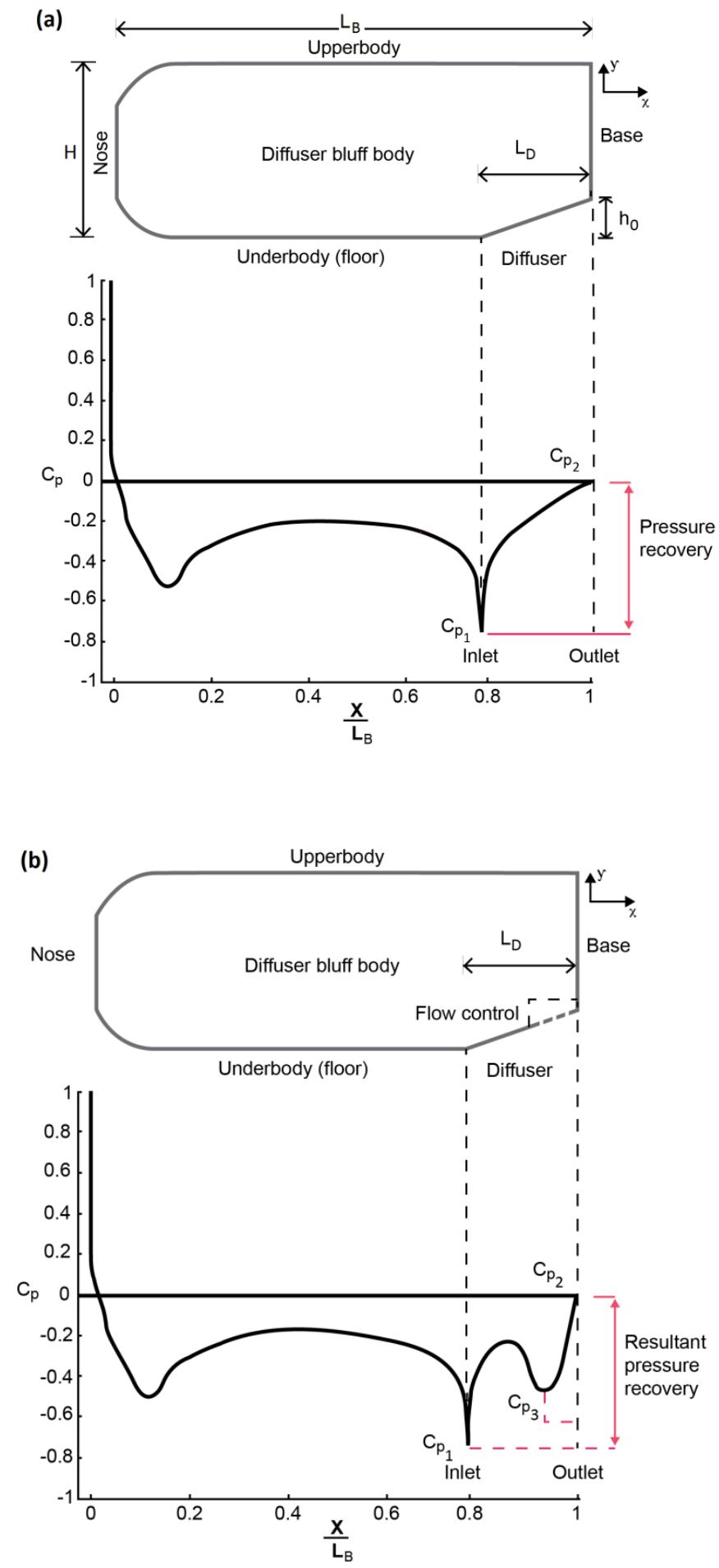

Figure 1: A schematic of the diffuser bluff body cross-section (adapted from [13]) showing: (a) Underbody center line surface pressure behavior of the bluff body highlighting a single pressure recovery at the diffuser section with no flow control, and (b) Underbody center line surface pressure behavior of the bluff body highlighting a second-stage pressure recovery at the diffuser section with flow control (in this case an inverted wing). 


\section{Experimental Setup}

\section{A. Wind Tunnel Test Facility}

Cranfield University's D.S. Houghton wind-tunnel was used for all testing. It is a $2.74 \mathrm{~m}$ by $1.66 \mathrm{~m}$ closedreturn, three-quarter open-jet wind tunnel equipped with a continuous-belt rolling road system. The movingground, synchronised with the tunnel freestream velocity, provided boundary layer suppression. The wind-tunnel ground boundary layer was removed by the application of suction immediately upstream of the rolling-road. The level and distribution of the suction were optimised during wind tunnel commissioning, and coupled with a knifeedge transition to the belt, ensured minimal belt boundary layer. The minimum recorded total pressure was $99.76 \%$ of the freestream value, occurring $0.75 \mathrm{~mm}$ above the surface of the belt, and a freestream turbulence intensity of $0.3 \%$. The action of the aerodynamic loads from the test vehicle were counteracted by the application of distributed suction to the underside of the rolling-road belt. The temperature of the air and rolling-road surface were held constant $\left(25^{\circ} \mathrm{C} \pm 0.5^{\circ} \mathrm{C}\right)$ throughout testing by active temperature control. Further details of the wind tunnel, rolling road and calibration are given by Knowles and Finnis [18]. Experimental results presented in this paper were obtained at a rolling road speed of $20 \mathrm{~ms}^{-1}$ and $U_{\infty}=20 \mathrm{~ms}^{-1}$, which corresponds to $\operatorname{Re}=1.8 \times 10^{6}$ (based on body length).

\section{B. Experimental Test Models}

Figure 2a shows a cross section schematic of the bluff body (baseline model), equipped with a $17^{\circ}$ diffuser ramp and in Figure $2 \mathrm{~b}$ it includes a wing close to the exit of the diffuser channel. The bluff body has the same dimensions as the body used by Senior $[8,9]$, which is $0.326 \mathrm{~m}$ in height, $0.314 \mathrm{~m}$ in width and $1.315 \mathrm{~m}$ long with $5 \mathrm{~mm}$-thick diffuser ramp side-plates. The wind tunnel blockage created by the model was $2.25 \%$. Figure $2 \mathrm{c}$ shows the model, which was made from aluminum and Sika Block polyurethane, mounted on an airfoil-shaped strut in the wind tunnel's test section. The inverted wing has an airfoil profile developed from a modified NASA GA (W) type LS (1)-0413 profile [19]. Coordinates for the pressure and suction sides of the airfoil profile are given by Ehirim [20]. Although only this airfoil profile was investigated, it was selected because it has been employed in several wing-in-ground effect studies [19, 21-23], and has a flat pressure surface (similar to the Clark $\mathrm{Y}$ airfoil profile) which provides a reasonably uniform offset from the diffuser ramp. The inverted wing was positioned across the diffuser channel, $14 \mathrm{~mm}$ away from the ramp surface, which is $\sim 50 \%$ of the estimated ramp boundary layer thickness (see Figure 3). It is held to the diffuser side plates by screws at both ends, close to the diffuser exit. The airfoil chord was set at $17^{\circ}$ to the $x$-axis to place the pressure surface in approximate alignment with the diffuser ramp. The chord to diffuser length ratio $\left(c / L_{D}\right)$ is 0.155 . 
(a)
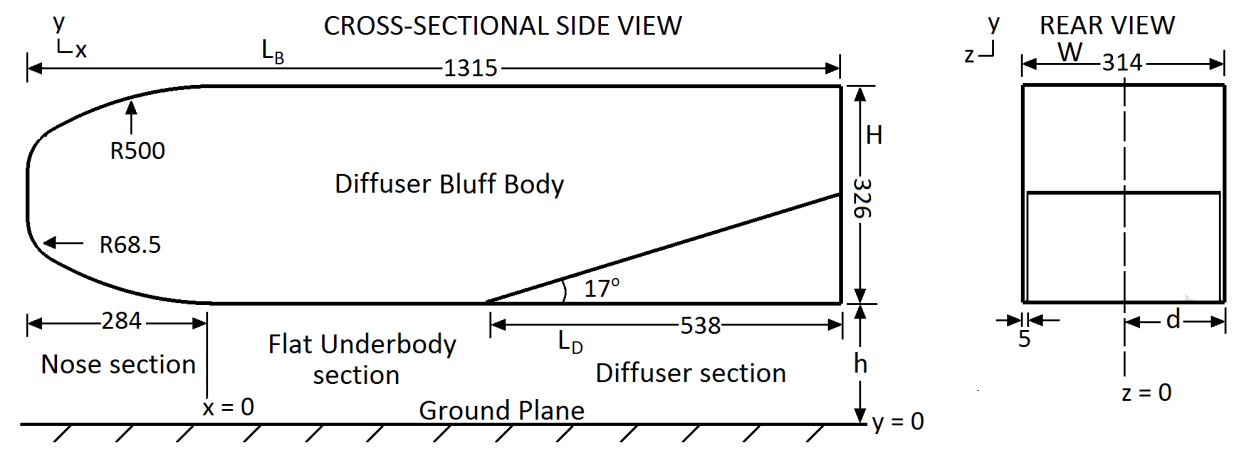

(b)

CROSS-SECTIONAL SIDE VIEW
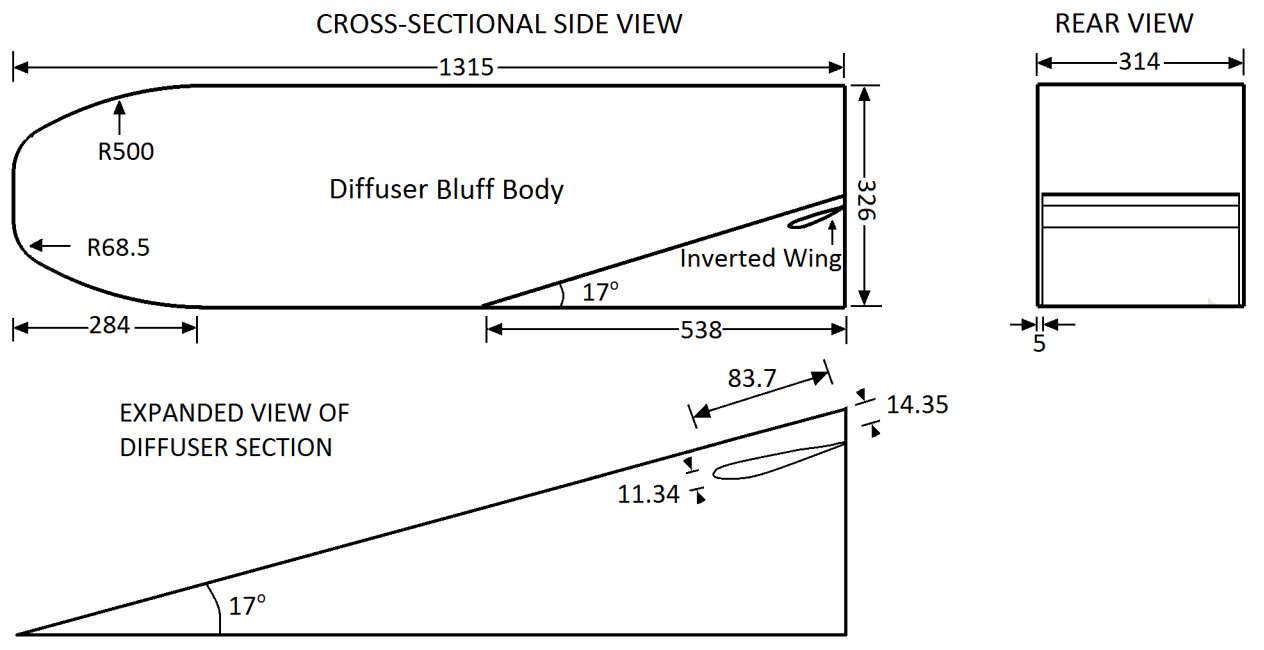

(c)

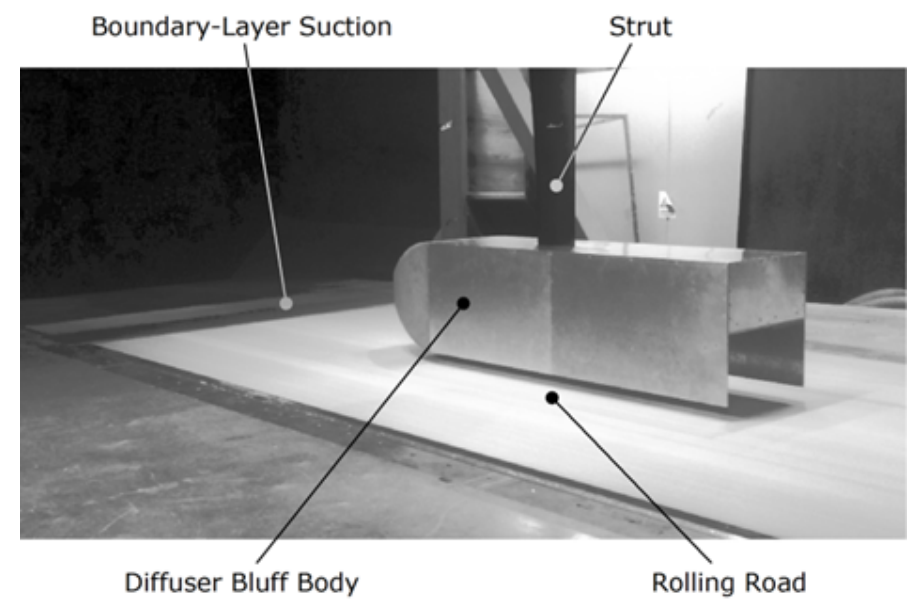

Figure 2: Details of the diffuser bluff body (dimensions in $\mathbf{m m}$ ) for: (a) plane diffuser, (b) diffuser with the wing, and (c) photograph of the diffuser bluff body model mounted on the strut in the Cranfield University DS Houghton wind tunnel 


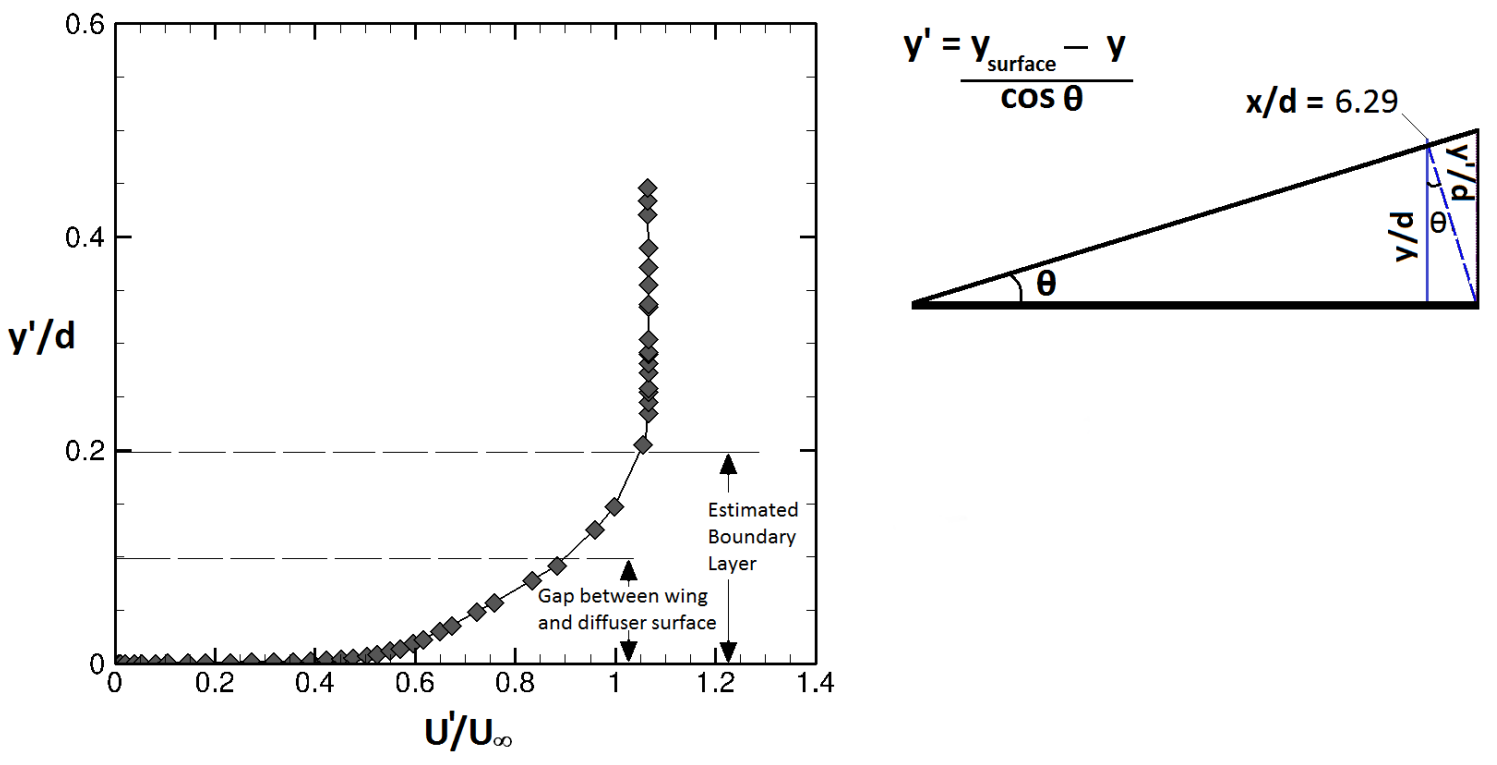

Figure 3: CFD-predicted boundary layer profile normal to the diffuser ramp surface for flow regime $\mathrm{A}(h / d=$ $0.764)$ with $y^{\prime} / d=0$ at the diffuser surface and positive downwards; $\mathrm{U}^{\prime} / \mathrm{U}_{\infty}$ is relative to the diffuser surface taken at $x / d=6.29$

\section{C. Experimental Methods}

The wind tunnel experiments were conducted at thirty-five different ride heights from $120 \mathrm{~mm}$ to $10 \mathrm{~mm}$ and, similar to the studies of Senior [8,9], ride height was non-dimensionalized by the half-width of the bluff body $d$ as $h / d=0.764$ to 0.064 . Half-width was chosen as the normalization parameter because the diffuser performance, in particular the position of the streamwise vortices, is dependent on the diffuser aspect ratio, derived from the ride height and the diffuser width. . A six-component force balance inside the model was used to measured time-averaged downforce and drag. Allowing for variation of air density due to changes in freestream pressure and temperature during the experiments, coefficients of downforce $C_{L}$ and drag $C_{D}$ were computed. Surface pressures were measured by seventy-one surface pressure tappings distributed on the flat and ramped underbody surfaces of the diffuser bluff body, as shown in Figure 4. The distribution includes eleven and twelve tappings positioned along the mid-line of the flat and ramp surfaces of the body respectively, with forty tappings distributed equally along four rows $(x / d=3.63,4.10,5.02,5.95)$. A fifth row $(x / d=6.29)$ of eight tappings was also distributed across the ramp surface above the wing. The thickness of the wing $(\sim 11 \mathrm{~mm})$ was too small to contain an adequate number of pressure tappings, hence equivalent $C_{p}$ data from CFD results along the wing suction surface center line and across $x / d=6.29$ are presented. Flow visualisation on the diffuser ramp surface was done with a paint based on a mixture of fluorescent pigment, oleic acid and paraffin.

Experimental repeatability was assessed by conducting non-consecutive test runs. Force coefficients, $C_{L}$ and $C_{D}$, and the pressure coefficient, $C_{p}$, were repeatable to $\pm 0.00010, \pm 0.00024$ and \pm 0.003 respectively. Force 
SAE Journal of Passenger Cars - Mechanical Systems

balance measurement errors in lift and drag were respectively $\pm 0.016 \%$ and $\pm 0.078 \%$ of full-scale based on their calibration. The model ride height was measured, using a drop-height gage, to an accuracy of $\pm 0.02 \mathrm{~mm}$ $\left(h / d= \pm 127 \times 10^{-6}\right)$, and pitch and yaw were set to within $\pm 0.04^{\circ}$ and $\pm 0.05^{\circ}$ respectively. Relative to fullscale, the respective measurement errors in the dynamic pressure and in the surface pressures was $\pm 0.20 \%$ and $\pm 0.25 \%$. Using the the root-mean-square procedure outlined in $[24,25]$, the total measurement uncertainties evaluated at a $95 \%$ confidence level for $C_{L}, C_{D}$ and $C_{p}$ were $\pm 0.0025, \pm 0.0032$ and \pm 0.057 respectively. Blockage correction was assessed using the method of Maskell [26], corrected for open-jet wind tunnels [27, pp. 425], which gave corrections to the force and pressure coefficients that were smaller than the uncertainty of the measurements. As a consequence, no blockage corrections have been applied to the data.

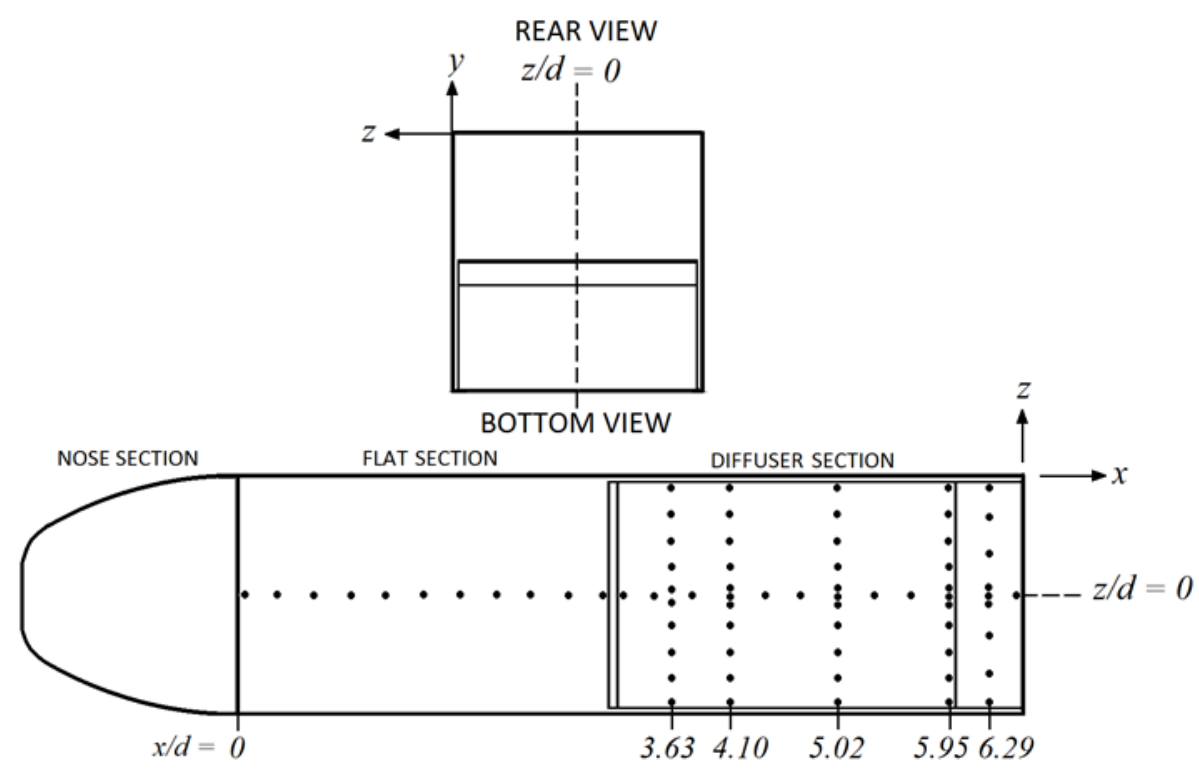

Figure 4: Distribution of pressure tappings on the diffuser bluff body

\section{Computational Methodology}

\section{A. Computational Grid and Domain}

A representation of the DS Houghton wind tunnel's test section was set up as the computational domain for the Computational Fluid Dynamics (CFD) simulations. Figure 5a shows the equivalent wind tunnel test section dimensions of the computational domain and the positional dimensions for the Computer Aided Design (CAD) model of the diffuser bluff body within the domain. The dimensions of the domain are similar to the test section of the wind tunnel and are given as: $1.66 \mathrm{~m}$ (height) by $2.74 \mathrm{~m}$ (width) by $6.0 \mathrm{~m}$ (length), while the positional distance of the bluff body within the domain is: $1.978 \mathrm{~m}$ (front); $2.707 \mathrm{~m}$ (rear) and $1.213 \mathrm{~m}$ (on each side). 
SAE Journal of Passenger Cars - Mechanical Systems

The surface and volume mesh for the domain was created using the commercial ANSYS ICEM CFD meshing software [28]. The mesh was made up of a hybrid grid of unstructured tetrahedral and structured prism layers as shown in Figure 5b. The maximum mesh dimension on the body was $0.006 \mathrm{~m}$ with $0.001 \mathrm{~m}$ for the wing and away from the body the maximum mesh dimension was $0.070 \mathrm{~m}$ (with a $0.050 \mathrm{~m}$ maximum for the rest of the moving ground). To improve the resolution of flow simulations, however, a 'virtual box' with a maximum mesh dimension of $0.010 \mathrm{~m}$ extending from the leading surface to the trailing surface of the domain was created around the model. The box is $0.689 \mathrm{~m}$ high and extends $0.163 \mathrm{~m}$ on each side of the body. The meshing strategy employed a $y^{+}$value of 0.7 (generating a first prism layer height of $\sim 1.2 \times 10^{-5} \mathrm{~m}$ ) with the surface mesh created by a tessellation of the CAD model surface and the volume mesh was generated by Delaunay triangulation.

The number of boundary layer prism layers, exponential growth of the layers and total grid size were dependent on the ride height simulated. Depending on the bluff body ride heights investigated $(h / d=$ $0.764,0.382,0.191,0.153$ and 0.064 ), the number of prism layers and total cell count varied from 15 to 33 and 27 million to 40 million respectively, with an exponential growth ratio ranging from 1.2 to 1.6. However, grid sensitivity of the computational solution was assessed using three different grids $(\sim 14, \sim 27$ and $\sim 50$ million cells) created at $h / d=0.191$ (maximum downforce ride height). The grid sensitivity of the force coefficients was noticeable between the coarse mesh of $\sim 14$ million cells and fine mesh of $\sim 27$ million cells, with a variation of about $2 \%$. When the fine mesh was refined further to about $\sim 50$ million cells, a much smaller difference between the force coefficients for the fine and refined grid was observed $(<1 \%)$. As a result, the need to conserve computational cost was prioritized and a minimum grid of $\sim 27$ million cells across the ride height test cases was used for this study. A domain sensitivity study was also conducted with the downstream boundary set at $\sim 2 L_{B}$, $\sim 6 L_{B}$ and $\sim 10 L_{B}$. The difference in the force coefficients between each domain was less than $1 \%$. To reduce computational cost the smaller domain size was chosen.

The open test section of the wind tunnel was simulated by setting up symmetry boundary conditions on the surrounding walls (the top and sides of the computational domain). This was done to create a zero-shear slip wall condition which mitigates wall boundary layer development and maintains a relatively constant static pressure across the walls. The domain's upstream boundary was defined as a velocity inlet condition with a freestream velocity of $20 \mathrm{~m} / \mathrm{s}\left(\mathrm{Re}=1.8 \times 10^{6}\right.$ by bluff body length) and a $0.01 \mathrm{~m}$ turbulence length scale which corresponds to the $\sim 0.3 \%$ turbulence intensity of the wind tunnel. An outlet gauge pressure of 0 Pa was specified for the downstream boundary with a $0.01 \mathrm{~m}$ length scale for the backflow. A no-slip moving wall condition was defined for the ground boundary with a translational velocity of $20 \mathrm{~ms}^{-1}$. Also, the diffuser bluff body within the domain was defined with a no-slip condition and as a stationary wall. 
(a)

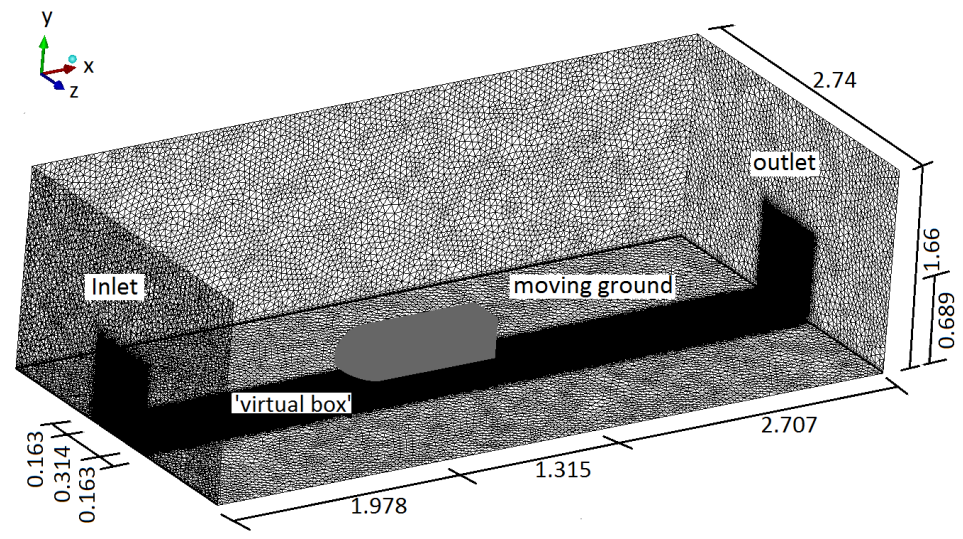

(b)

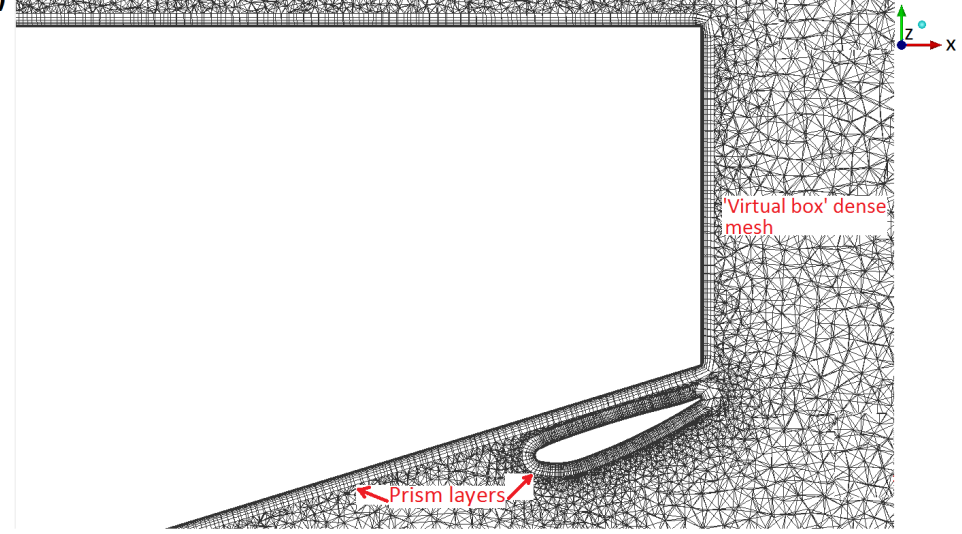

Figure 5: CFD grid. (a) An illustration of the computational domain dimensions (in $\mathrm{m}$ ) representing the wind tunnel test section. (b) A close-up view of the central plane cross section of the computational domain highlighting the mesh distribution around the bluff body and wing.

\section{B. Numerical Methods}

The ANSYS FLUENT solver [29] was used for the CFD simulations. The two-equation $\kappa-\omega$ SST turbulence model [30] was used for the steady Reynolds-Averaged Navier-Stokes (SRANS) simulations for $h / d=$ 0.764 and 0.382 . At lower ride heights, however, laminar to turbulent transition around the nose section (near the location where it merges with the flat section) of the bluff body model increasingly influenced the downstream diffuser flow. Therefore, the $\gamma-R e_{\theta}$ (Gamma-Re-theta) transitional SST turbulence model [31, 32] was used for $h / d=0.153$ (with an intermittency factor set at 0.46 , where 0 and 1 represent fully laminar and fully turbulent flow respectively) and the $k-k l-\omega$ transition model [33] was employed for $h / d=0.191$ and $0.064\left(\right.$ with $y^{+}=$ 0.1 generating a first prism layer height of $1.7 \times 10^{-6} \mathrm{~m}$ ). After convergence of the force coefficients was reached over 10,000 iterations with the residuals dropping below $10^{-5}$, transient simulation was initialized from the inlet boundary. In the case of $h / d=0.191$ and 0.064 , the transient simulations were done using the unsteady Reynolds-Averaged Navier-Stokes (URANS) mode of the $k-k l-\omega$ transition model. The transient cases 
SAE Journal of Passenger Cars - Mechanical Systems

of $h / d=0.764,0.382$, and 0.153 , however, were done with the coupling of the Detached Eddy Simulation (DES) approach and the various transitional RANS turbulence models. The standard version of DES employs a hybrid RANS/LES (large eddy simulation) approach, which models the near-wall region of the flow with RANS and the far-wall flow regions with LES $[34,35]$. A deficiency with the standard version is its tendency to transition much faster to LES mode even in areas of the boundary layer that require RANS mode. As a result, the DelayedDES (DDES) approach was developed by Spalart et al. [36] as a modification of the standard DES and the update involved the addition of blending functions to the $\kappa-\omega$ SST governing formulations to mitigate the abrupt transition tendencies within the boundary layer. Despite these modifications, log-layer mismatch occurred at boundary locations between the RANS and LES regions. Consequently, an advanced methodology known as the Improved Delayed Detached Eddy Simulation (IDDES) was developed [37, 38]. It combines the strengths of DDES and wall-modelled LES with the inclusion of empirical and elevating functions to curb log-layer mismatch and grid-induced separation. This enhanced competency of the IDDES approach made it appropriate for use in the CFD investigations of this study.

\section{C. Simulation Strategy}

The incompressible flow within the computational domain was solved with the implicit pressure-based solver using the 3-D finite volume method. For the SRANS methodology, the discretization for the convective and viscous terms made use of a second-order upwind scheme. Also, the spatial discretization of the pressure term was implemented using the standard interpolation scheme with the Green-Gauss node-based scheme used to compute cell gradients. The Semi-Implicit Method for Pressure Linked Equations (SIMPLE) algorithm was employed to couple the pressure-velocity fields. For the IDDES methodology, the momentum term was implemented with the bounded central differencing scheme and the implicit, second-order scheme used for the temporal discretization. The standard interpolation scheme was employed for the pressure term with the GreenGauss node-based scheme used for the computation of cell gradients. In addition, the bounded second-order implicit method was used for the transient formulation with the pressure-velocity fields coupled with the Pressure Implicit with Splitting of Operator (PISO) scheme.

Using the guidelines defined by Spalart [39], a time-step of $\Delta_{t}=3.33 \times 10^{-5} \mathrm{~s}$ based on freestream velocity $U_{\infty}$ and smallest $x$-wise grid size $\Delta_{x}=0.001 \mathrm{~m}$ (where $\Delta_{t} \approx \Delta_{x} / 1.5 U_{\infty}$ ) was employed for the simulations. As a result, the convergence of the numerical approximations of the finite-volume partial differential equations was achieved with a Courant-Friedrichs-Lewy (CFL) number criterion of $C F L \leq 1$, where $C F L \approx U_{\infty} \Delta_{t} / \Delta_{x}$. The flow required a simulation time of $3 \mathrm{~s}$ to adequately develop, which equates to 90,091 time-step iterations 
SAE Journal of Passenger Cars - Mechanical Systems

with a residual convergence criterion of 3-decimal places accomplished within 20 sub-iterations. Over the course of the flow simulation time, the flow particle traveling the length of the bluff body at freestream velocity completes 900 passes. After the residuals, force and surface pressure coefficients of the transient flow had reached a pseudosteady state, statistical data (mean) were then collected. The simulations were done on the Cranfield University DELTA high performance computing (HPC) cluster with each simulation completed within 140 hours (wall clock time) using 256 cores on the system.

\section{Results}

The directly proportional relationship between $C_{L}{ }^{*}$ and $C_{D}$ is confirmed in the plots of Figure 6 (for the baseline diffuser with a plane ramp surface) where an increase in downforce corresponds with a similar increase in drag and vice versa. Also, Figures $6 \mathrm{a}$ and $6 \mathrm{~b}$ show that reducing the ride height causes an increase in downforce and drag until a maximum is reached after which subsequent ride height reductions lead to decreases in downforce and drag.

Following Senior [8, 9] four flow regimes are identified across the ride height interval and classified as: force enhancement (flow regime A), maximum force (flow regime B), force reduction (flow regime C) and low force (flow regime D). The rationale for the location of these regimes was discussed by Ehirim et al. [40]. Region A is distinguished by a reasonably symmetrical diffuser flow due to the presence of a pair of longitudinal vortices. Region B is characterised by increased inlet suction and vortex pair size. Region $\mathrm{C}$ is where the diffuser flow becomes asymmetric, and region $\mathrm{D}$ is where the asymmetric flow is increasingly dominated by flow recirculation. Throughout this paper, as highlighted in Figures $6 \mathrm{a}$ and $6 \mathrm{~b}$, regime $\mathrm{A}$ is represented at $h / d=0.764$ and 0.318 ; regime $\mathrm{B}$ is represented at $h / d=0.191$; regime $\mathrm{C}$ is represented at $h / d=0.153$ and regime $\mathrm{D}$ is represented at $h / d=0.064$.

\footnotetext{
${ }^{*}$ Throughout this paper, $-C_{L}$ represents downforce.
} 


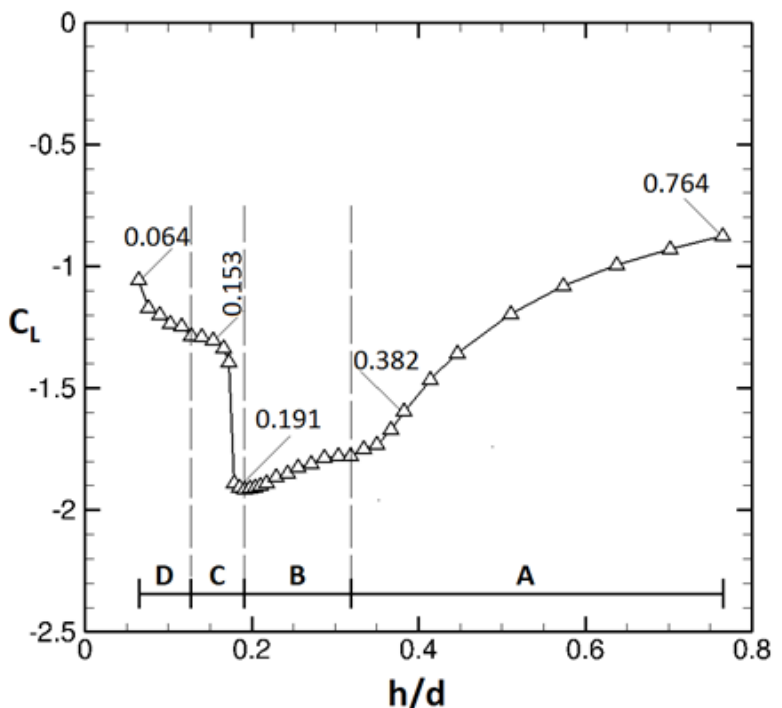

(a)

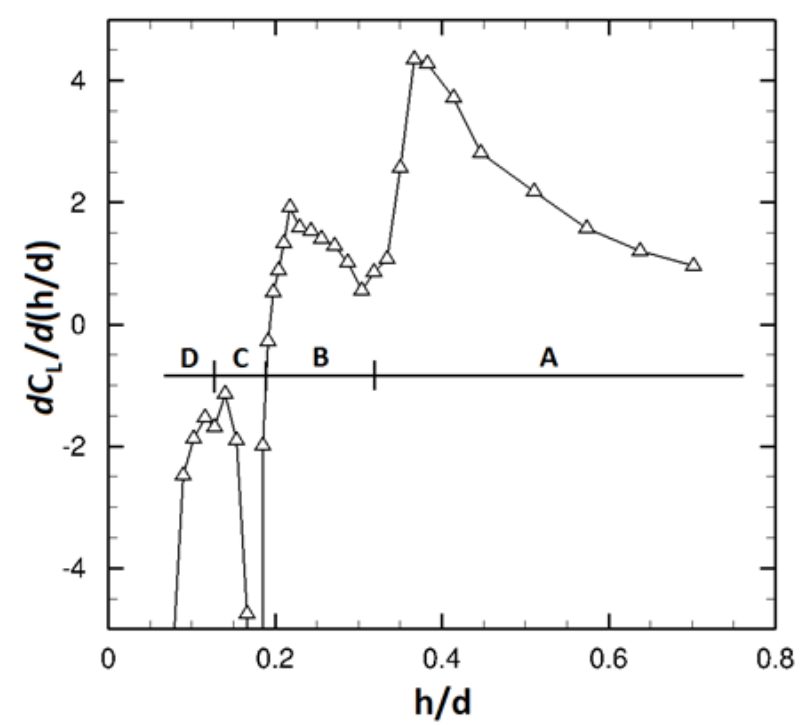

(c)

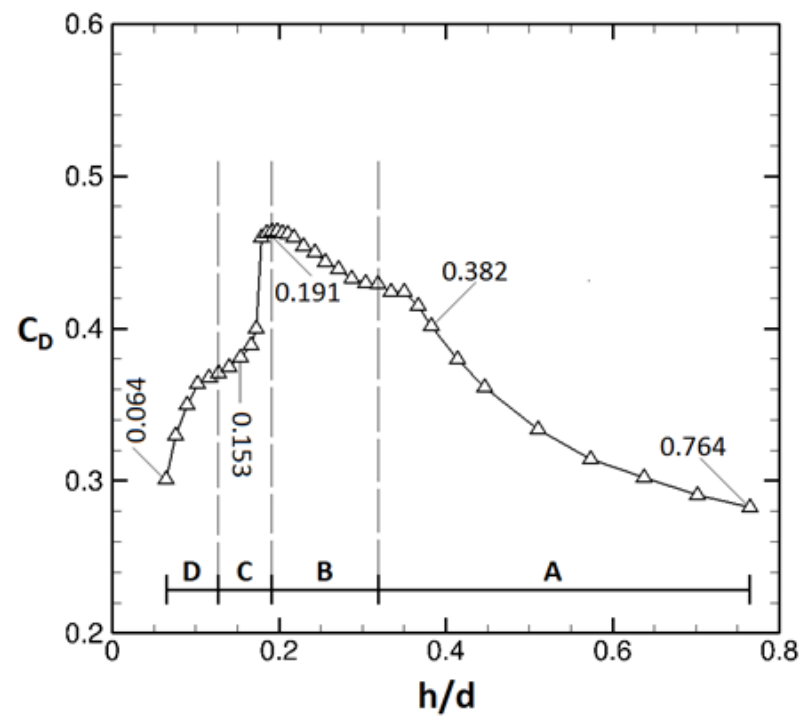

(b)

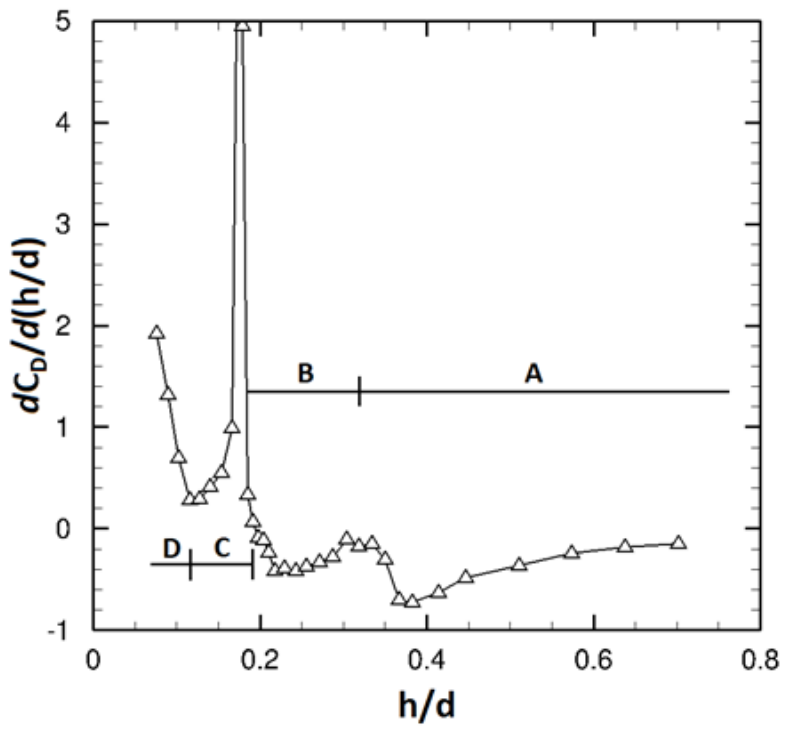

(d)

Figure 6: Wind tunnel force measurements across ride heights ( $h / d=0.764$ to 0.064 ) and flow regimes (' $A$ ' to ' $D$ ') for plane diffuser: (a) lift coefficient (b) drag coefficient, (c) lift coefficient slope (d) drag coefficient slope

An inverted wing is applied within the diffuser (as shown in Figure 7) as a passive flow control device to induce a second-stage pressure drop and recovery region close to the diffuser exit. The occurrence of the secondary pressure recovery region close to the diffuser exit lowers average static pressure before the flow exits the diffuser at the higher atmospheric pressure. As a result of this action downforce is increased. Notably, the gap between the pressure surface (top side) of the wing and the ramp surface is critical to the performance of the inverted wing as a passive flow control device. This is because the diffusing flow of the diffuser follows the trajectory of the upwardly-inclined diffuser ramp. Hence, if the wing is not at an angle of attack $(\alpha)$ the same as the diffuser angle $(\theta)$, the wall-bounded airflow between the diffuser ramp and the wing separates from the pressure surface of the wing. As a consequence, downforce reduces and aerodynamic drag increases. 


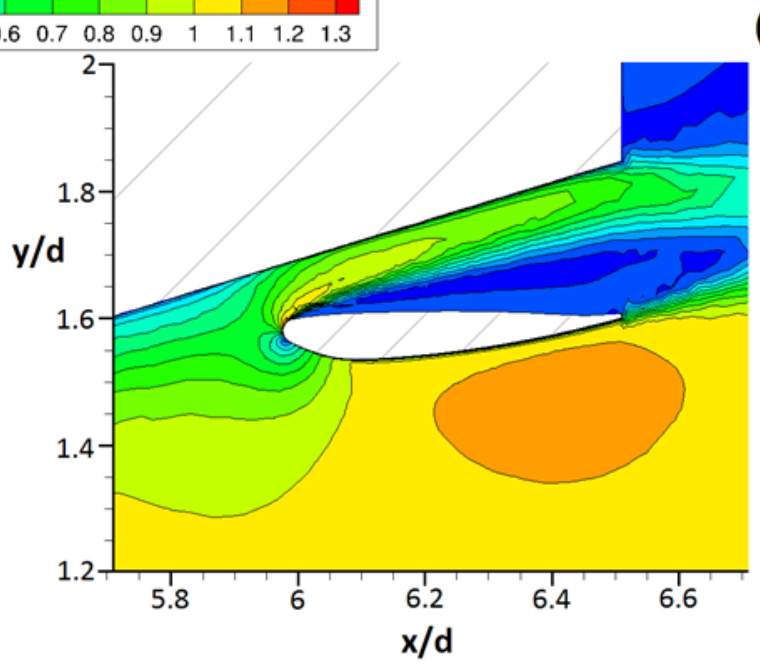

(c)
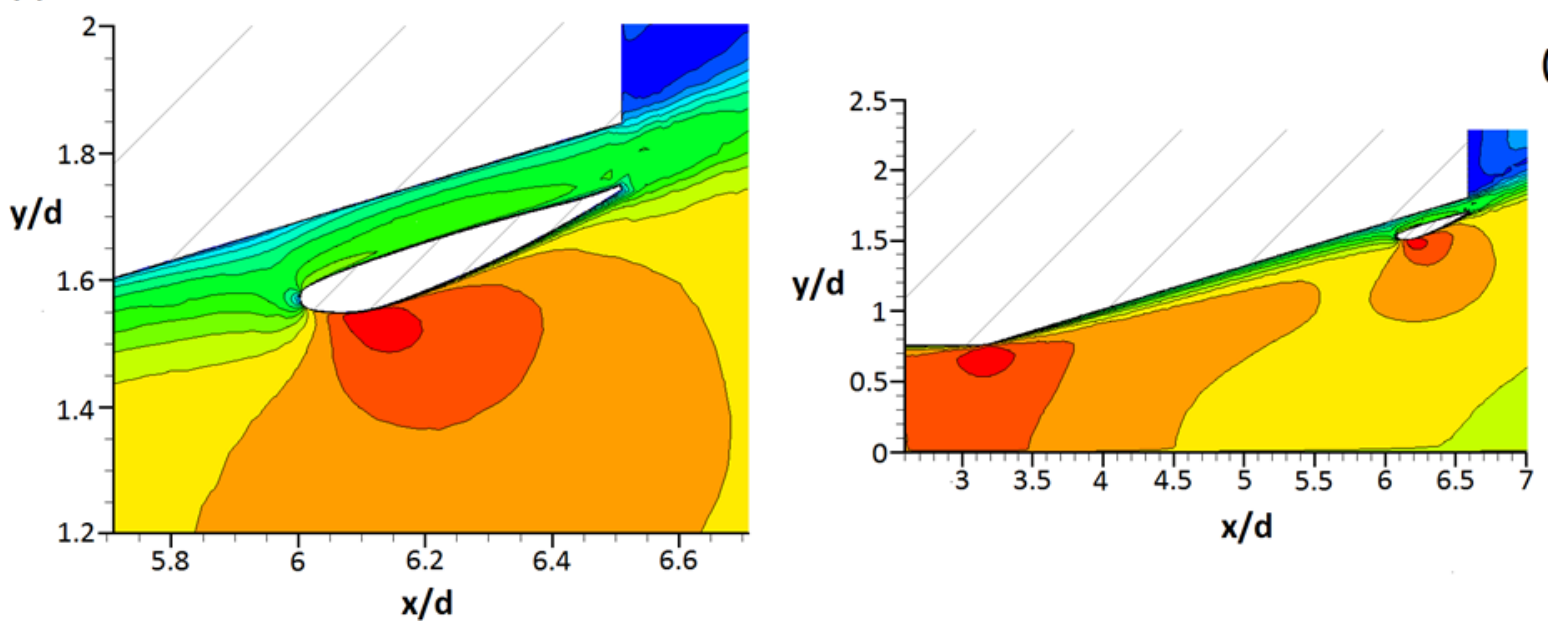

Figure 7: Magnified plot scale of CFD total velocity $U$ contours on centerline plane $(z / d=0)$ near the diffuser exit of the bluff body at $h / d=0.764$ for: (a) plane diffuser (b) diffuser with an inverted wing at $\alpha=0^{\circ}$ (c) diffuser with an inverted wing at $\alpha=17^{\circ}$, and (d) an expanded contour plot scale showing diffuser inlet to exit for diffuser with an inverted wing at $\alpha=17^{\circ}$

Preliminary investigations with CFD indicated that at $\alpha=0^{\circ}$ (Figure 7b), airflow did, indeed, separate from the pressure surface of the inverted wing producing a reduction in $C_{L}$ and $C_{D}$ at $h / d=0.764$ of $\sim 18 \%$ and $\sim 14 \%$, respectively, relative to the coefficients predicted for the baseline plane diffuser (Figure 7a). Also the flow velocity around the leading edge of the wing appeared to have reduced because the wing was not aligned with the local flow direction, therefore inhibiting the higher flow velocities induced by the suction surface of the wing when $\alpha=17^{\circ}$ (Figure 7c). As a result, downforce was reduced, however, drag also reduced due to the loss of downforce induced by the separated flow above the wing — thus decreasing the accompanying lift-dependent drag. In Figure 7c, where $\alpha$ was increased to $17^{\circ}$ (the same as the diffuser angle), flow separation over the pressure surface is removed, leading to an increase in $C_{L}$ of $\sim 39 \%$ and an increase in $C_{D}$ of $\sim 23 \%$ relative to the zero angle of attack values. Also, as shown in Figure 7d, the suction side of the wing induced the second-stage pressure drop and recovery by accelerating the flow near the diffuser exit to velocities similar to those seen at the diffuser inlet. 


\section{A. Force Measurements}

In Figure 8, the ride height interval between $h / d=0.764$ and 0.318 makes up regime A. In this region, there is a monotonic increase in both downforce and drag and as a result it is denoted as the force enhancement region. At the highest ride height of the interval $(h / d=0.764)$, the modified diffuser increased the values of $C_{L}$ and $C_{D}$ from those of the plane diffuser by $12 \%$ and $11 \%$ respectively while at $h / d=0.318$, it increased $C_{L}$ and $C_{D}$ by $3.1 \%$ and $4.2 \%$ respectively.

Subsequently, in regime B, which falls within the interval $h / d=0.318$ to 0.191 , a further increase in $C_{L}$ and $C_{D}$ occurred with a change in the gradient for the $C_{L}$ and $C_{D}$ curves. In the $C_{L}$ and $C_{D}$ plots for the plane diffuser in Figure 8, the inflection around $h / d=0.318$ indicates that a change in the diffuser flow regime has occurred. However, the observation is more distinct for the case of the modified diffuser, where the inflection around $h / d=0.318$ is preceded by a flattening of the $C_{L}$ and $C_{D}$ curves. At the maximum force ride height of $h / d=0.191$, the modified diffuser increased $C_{L}$ and $C_{D}$ by $3.0 \%$ and $1.9 \%$ respectively.

Regime C occurs within the interval of $h / d=0.191$ to 0.127 (Figure 8 ) and the steep drop in the slope preceded by the inflection of the $C_{L}$ and $C_{D}$ curves around $h / d=0.191$, indicated the occurrence of another change in the diffuser flow. Relative to the plane diffuser, the modified diffuser with the inverted wing, increased $C_{L}$ by about $2.4 \%$ at $h / d=0.153$.

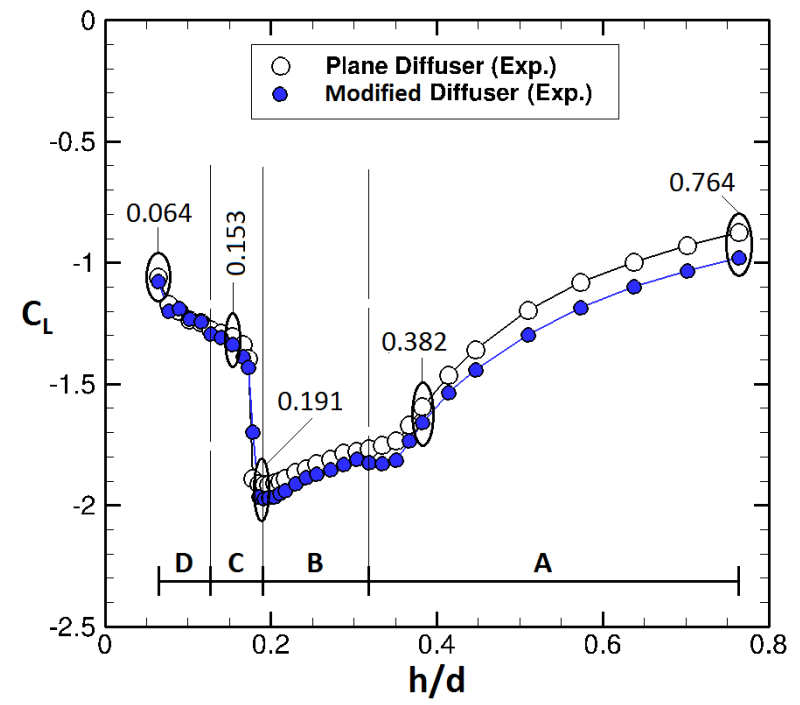

(a)

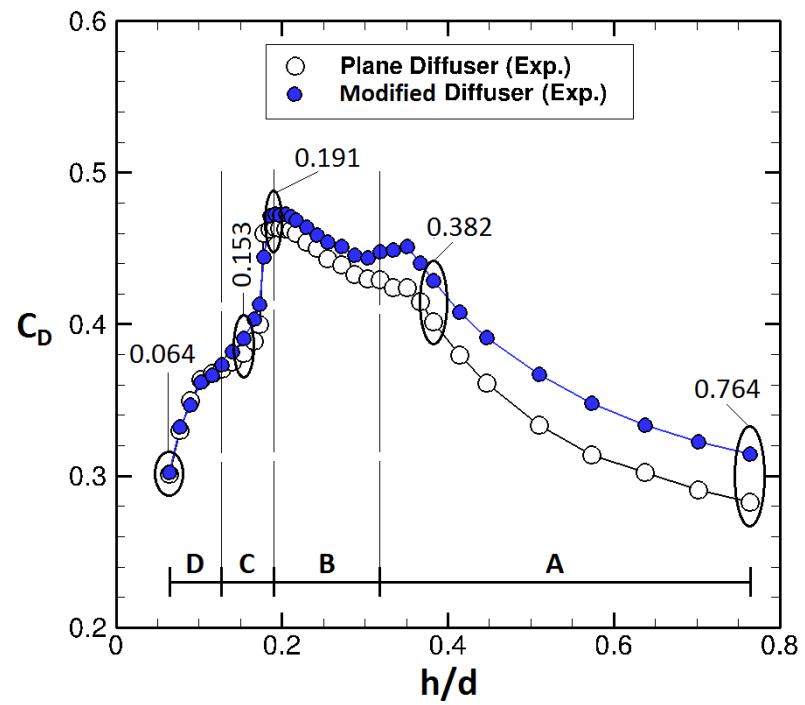

(b)

Figure 8: Wind tunnel force measurements coefficients across the range of ride heights investigated for the plane diffuser and modified diffuser (with the wing): (a) $C_{L}$; (b) $C_{D}$, highlighting the various flow regimes (from ' $A$ ' to ' $D$ ') and representative ride heights 
In regime $\mathrm{D}(h / d=0.127$ to 0.064$)$, force reduction continued to occur. The inflection of the $C_{L}$ and $C_{D}$ curves after $h / d=0.127$ implied that a change in the diffuser flow has occurred. At $h / d=0.064$, the modified diffuser increased the $C_{L}$ relative to that of the plane diffuser by about $1.9 \%$.

The percentage changes in downforce and drag are shown in Figure 9. Increase in downforce leads to a similar increase in drag, thus, as shown in Figure 9, the percentage differences in downforce correspond to similar percentage differences in drag. The percentage change in $C_{L}$ and $C_{D}$ between the modified and plane diffuser, is highest at the type A flow regime ride height of $h / d=0.764$. The modified diffuser generally generated an increase in downforce across the ride heights even though the percentage increase in force coefficients gradually reduces from flow regime A to $\mathrm{B}$. However, as a result of the severe drop in downforce and drag at the forcereduction (type $\mathrm{C}$ regime) ride height of $h / d=0.178$, there is a correspondingly large negative change at that ride height.

Figure 10 shows the lift-to-drag ratio and its percentage change between the modified and plane diffusers. It can be seen that the modified diffuser gives very little overall change in $L / D$ across the ride heights. In general, there is a slight reduction over much of regime A and half of regime B. Furthermore, peak L/D occurs at the maximum-force ride height of $h / d=0.191$ (flow regime B). This means that at a high-downforce aerodynamic setup, a racing car with the modified diffuser will have an increased aerodynamic "efficiency" (higher L/D), as well as an enhanced downforce.

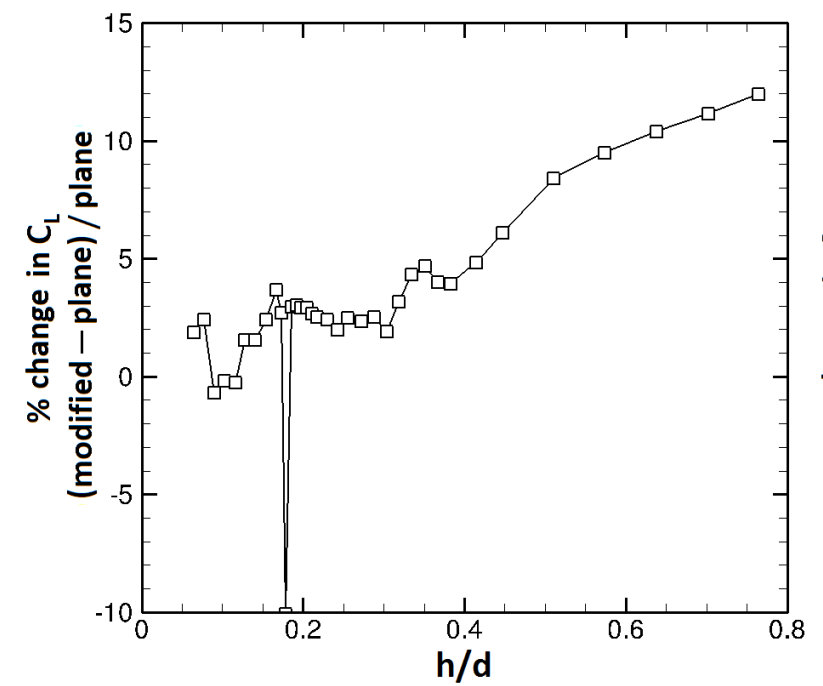

(a)

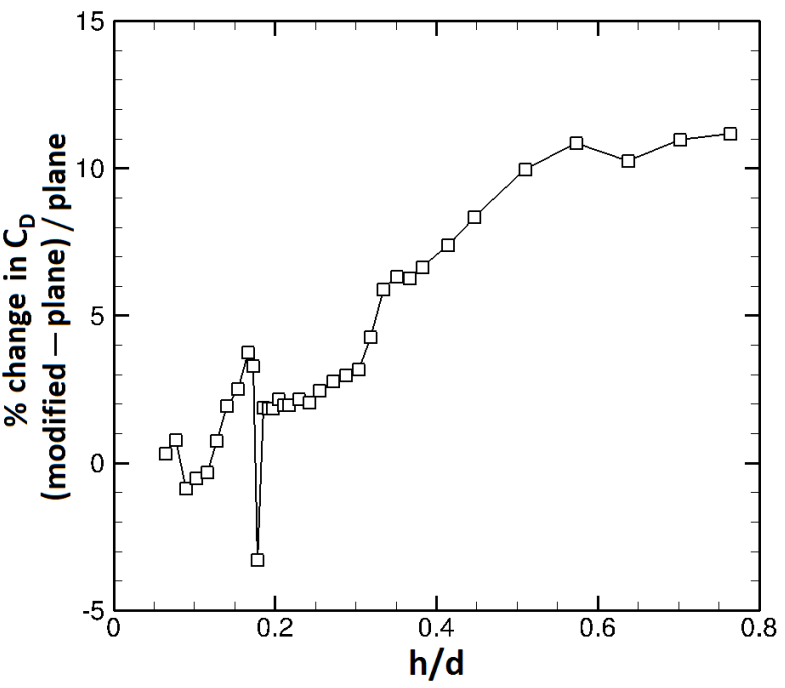

(b)

Figure 9: Percentage difference in force coefficients across the range of ride heights $(h / d=0.764$ to 0.064$)$ between the plane diffuser and the diffuser with the wing for: (a) $C_{L}$; (b) $C_{D}$ 


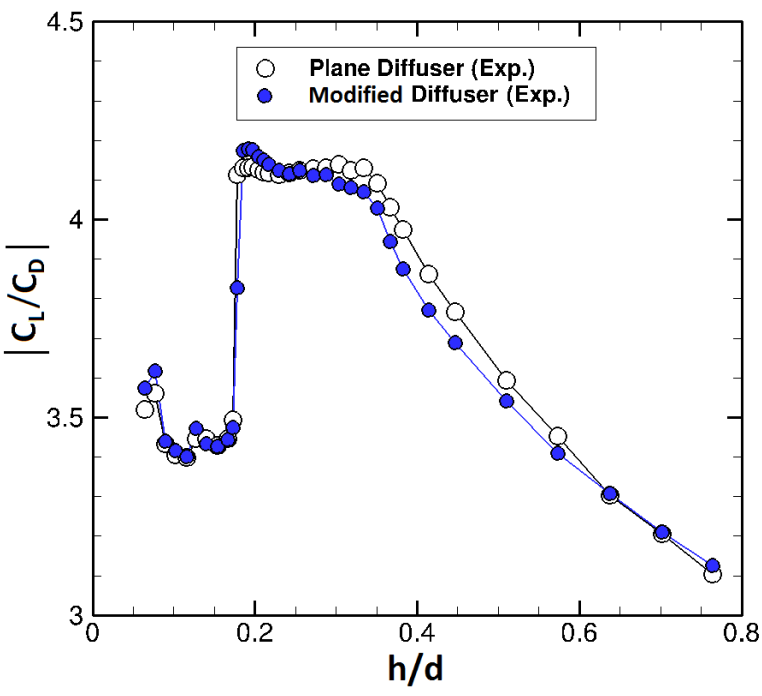

(a)

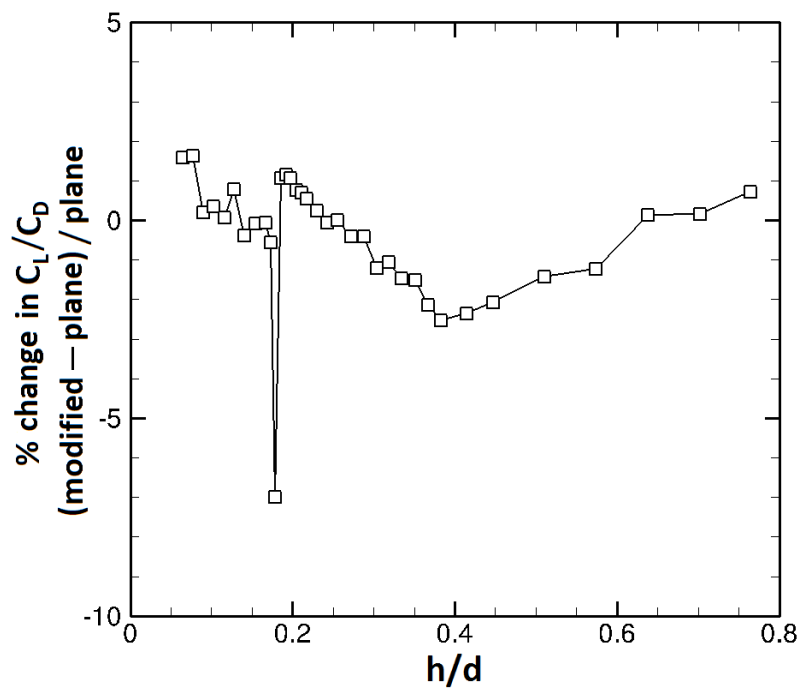

(b)

Figure 10: (a) Lift-to-drag ratio measured across the range of ride heights ( $h / d=0.764$ to 0.064 ) investigated for the plane and modified diffusers; (b) Percentage difference in lift-to-drag ratio between the plane and modified diffusers across the range of ride heights investigated

\section{B. Surface Pressures}

Surface pressures on the flat underbody and diffuser ramp surfaces of the wind tunnel test body were measured to determine the effects of the diffuser flow. Surface pressures for the suction side of the inverted wing were computed using CFD. The surface pressure distributions are presented as $C_{p}$ plots at ride heights that represent each of the four distinct force regimes $(h / d=0.764,0.382,0.191,0.153$, and 0.064$)$. Centerline underbody surface pressures are presented in Figure 11, whilst Figure 12 shows spanwise distributions of diffuser surface pressure.

As presented in Figure 11, the measured peak suction at the diffuser inlet is the same for both diffusers and the suction peak increased from that of the force enhancement regime ride height of $h / d=0.764$ to that of the maximum force ride height of $h / d=0.191$. The diffuser inlet peak suction then reduced to the low force regime ride height of $h / d=0.064$. This behavior signified that the flow velocity of the constrained flow underneath the test body (and the diffuser inlet peak velocity) increased from $h / d=0.764$ to $h / d=0.191$ but reduced from $h / d=0.191$ to $h / d=$ 0.064. It also indicated that the suction peak at the diffuser inlet influenced the pressure recovery downstream of the inlet. The CFD simulations generally predicted the correct shape of the pressure distributions but have under- or overpredicted the peak suction at the diffuser inlet.

Downforce increased in the case of the modified diffuser because, as shown in Figure 11, it induces a second-stage pressure recovery towards the diffuser exit. The inverted wing changed the local flow behavior close to the diffuser exit. Although the presence of the inverted wing increased (made less negative) the pressure on the diffuser ramp, the pressures on the suction surface of the wing (as indicated by the diamond symbols in Figure 11) are significantly lower than at the same location on the diffuser ramp. As a result, the modified diffuser generated more downforce than the plane diffuser. 
SAE Journal of Passenger Cars - Mechanical Systems

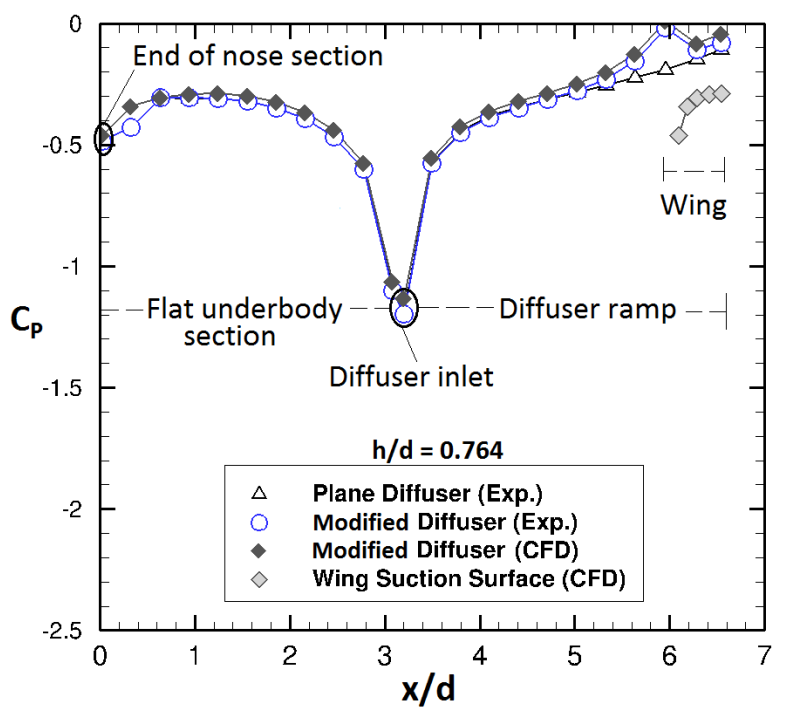

(a)

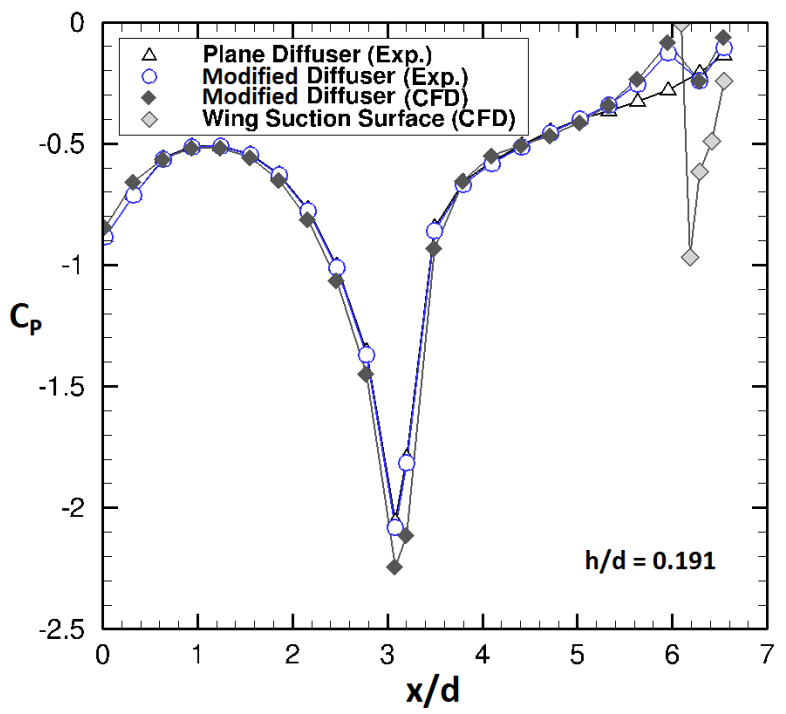

(c)

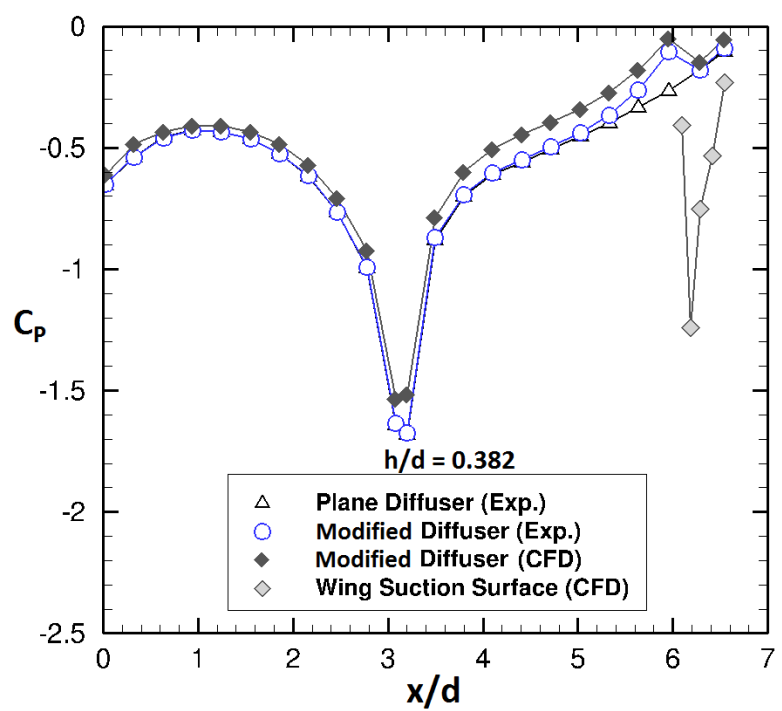

(b)

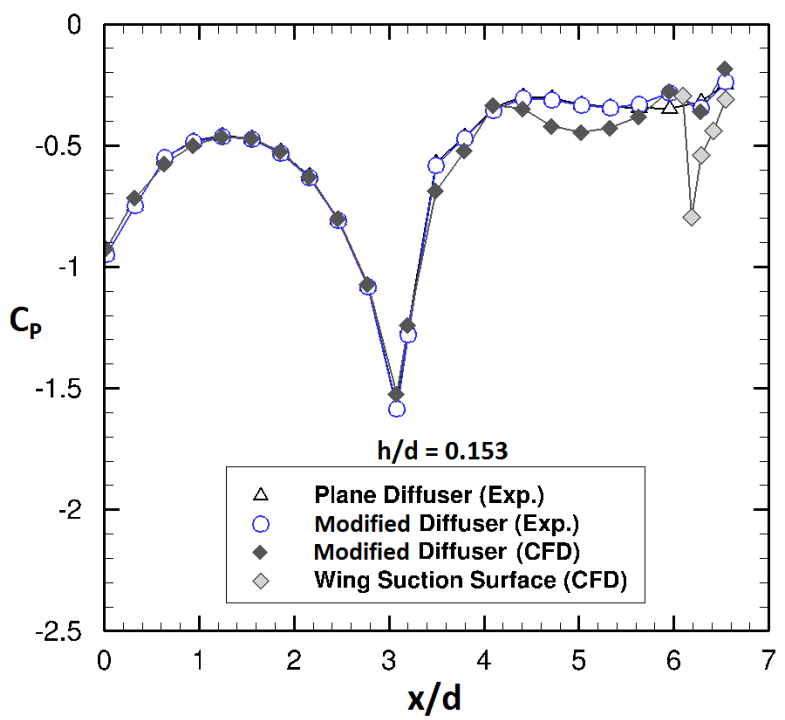

(d)

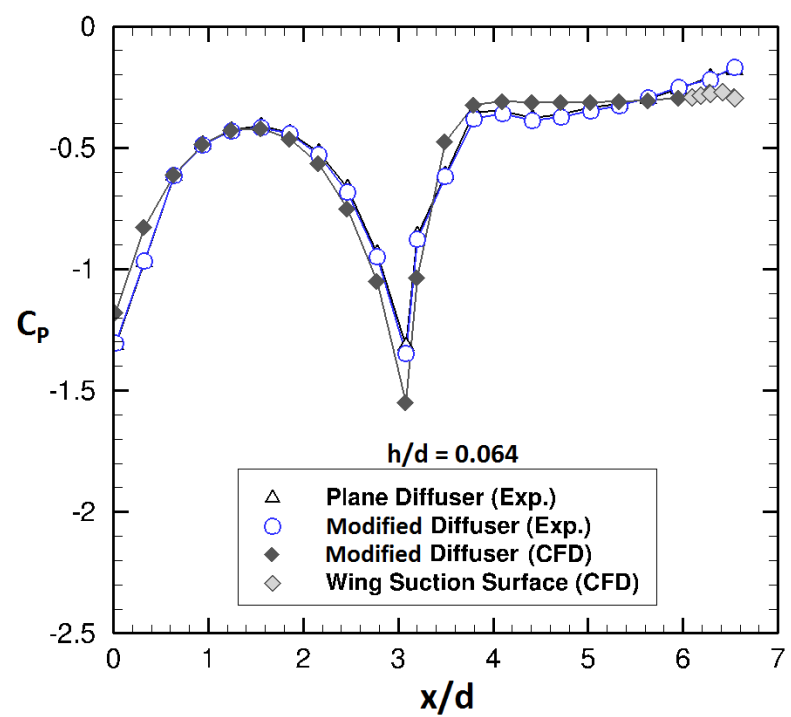

(e)

Figure 11: Measured and predicted underbody surface pressure distributions on centerline $(z / d=0)$ for plane diffuser and diffuser with the wing at: (a) $h / d=0.764$ (b) $h / d=0.382$ (c) $h / d=0.191$ (d) $h / d=0.153$ (e) $h / d=0.064$ 
SAE Journal of Passenger Cars - Mechanical Systems

In Figure 12a, it can be seen that the plane diffuser flow has significant spanwise variation near the inlet, where the end-wall vortices are growing, but more uniform flow by the time the exit is reached. In addition, the 3-D diffuser flow is symmetric in the force-enhancement and maximum-force regimes, represented by $h / d=$ $0.764,0.382$, and 0.191 (Figures $12 \mathrm{~b}$ to $12 \mathrm{~d}$ ). In contrast, the flow is asymmetric in the force-reduction and low-force regimes (Figures 12e and 12f). The spanwise pressures for the plane and modified diffusers decreased from the surface pressures at $h / d=0.764$ to the corresponding pressures at $h / d=0.191$. In contrast, the surface pressures increased from those at $h / d=0.191$ to the corresponding pressures at $h / d=0.064$.

Figure 12 also indicates that the CFD simulations accurately predict the general shape of the spanwise pressures on the diffuser ramp surface close to the exit $(x / d=6.29)$ even though there is a variation in the magnitude between the CFD predictions and wind tunnel measurements. The increase in downforce with the use of the wing is highlighted by the CFD predictions of significantly lower pressure on the wing suction surface at $x / d=6.29$ in comparison with plane diffuser surface pressures at the same location (Figure 12). The lower pressures on the wing generate the increased download seen in the force measurements. Flow symmetry is reflected in the pressure distributions on the wing suction surface at the force-enhancement (Figure 12b and 12c) and maximum-force ride heights (Figure 12d), but at the force-reduction (Figure 12e) and low-force ride heights (Figure 12e) flow asymmetry is observed. On-surface flow features that further explain the diffuser 3-D flow are presented in the next section. 
SAE Journal of Passenger Cars - Mechanical Systems

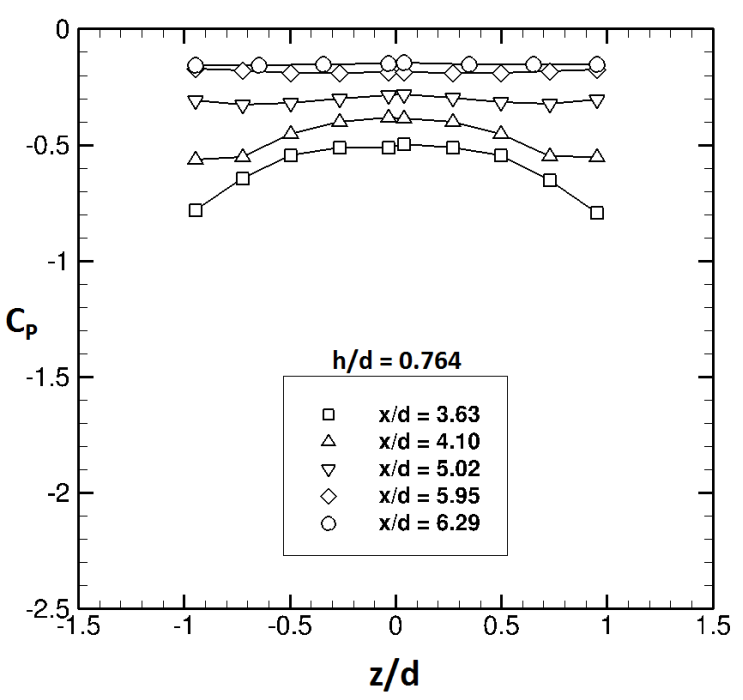

(a)

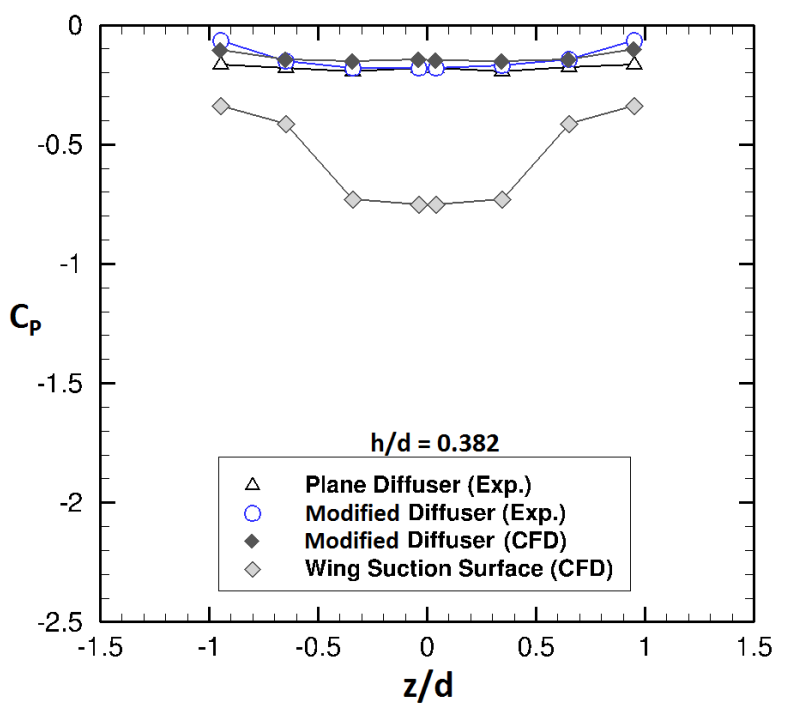

(c)

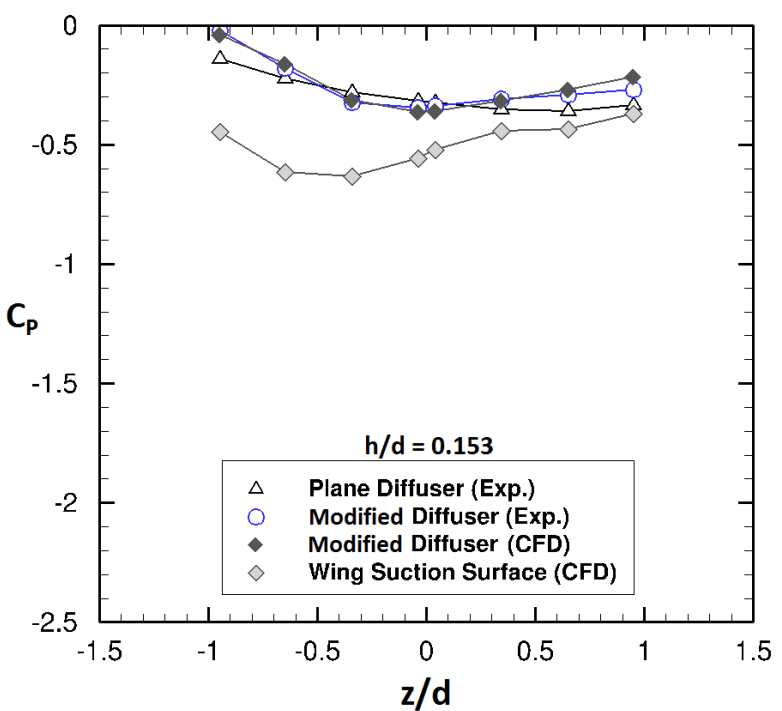

(e)

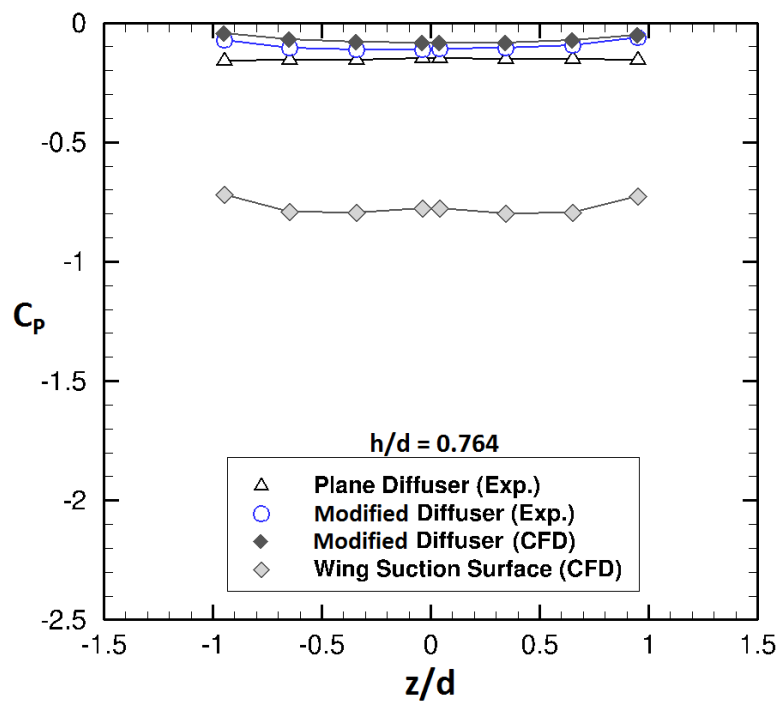

(b)

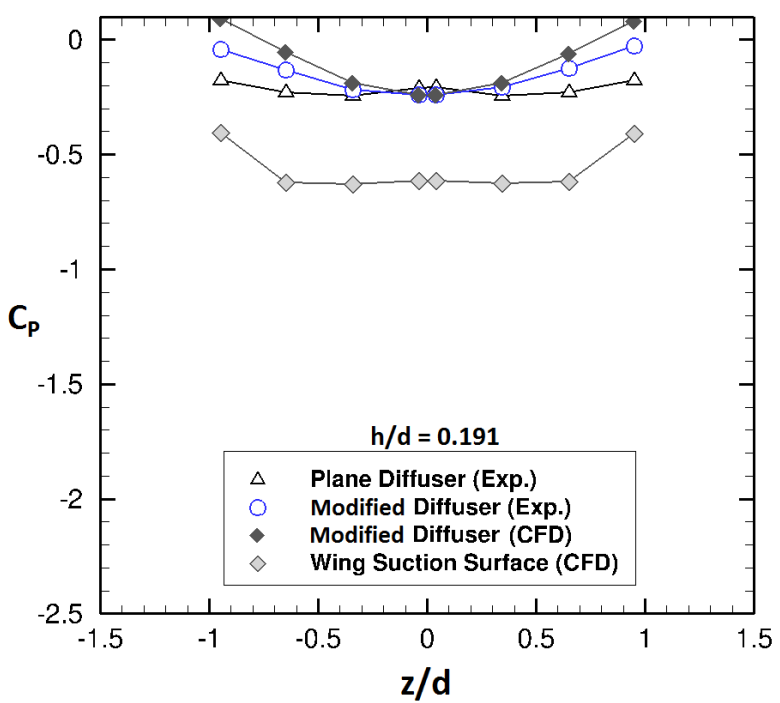

(d)

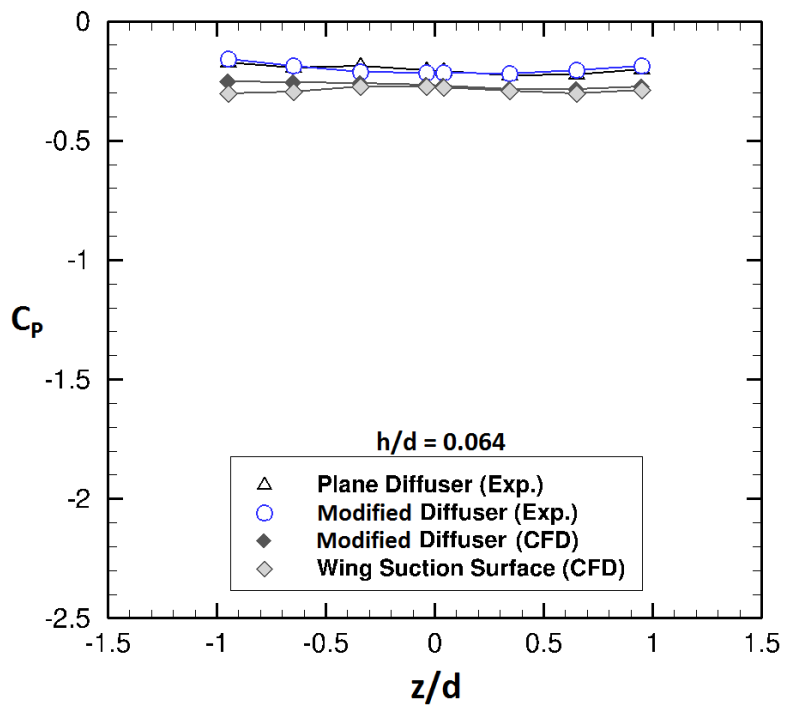

(f)

Figure 12: Spanwise surface pressure distributions. (a) Measured pressures on $x / d=3.63,4.10,5.02$, 5.95 and 6.29 for plane diffuser at $h / d=0.764$. Measured and predicted diffuser pressures on $x / d=$ 6.29 for plane diffuser and diffuser with the wing at: (b) $h / d=0.764$ (c) $h / d=0.382$ (d) $h / d=0.191$ (e) $h / d=0.153$ (f) $h / d=0.064$ 
SAE Journal of Passenger Cars - Mechanical Systems

\section{C. Surface Flow Visualisation}

The surface flow visualisation shown in Figures 13 to 17 illustrates flow features on the ramp surface of the plane and modified diffusers. The surface flow patterns on both diffusers are generally identical up until the region towards the diffuser exit, where the inverted wing is located in the case of the modified diffuser. The difference in the flow features at that location provides an insight into the downforce-enhancing characteristics of the modified diffuser.
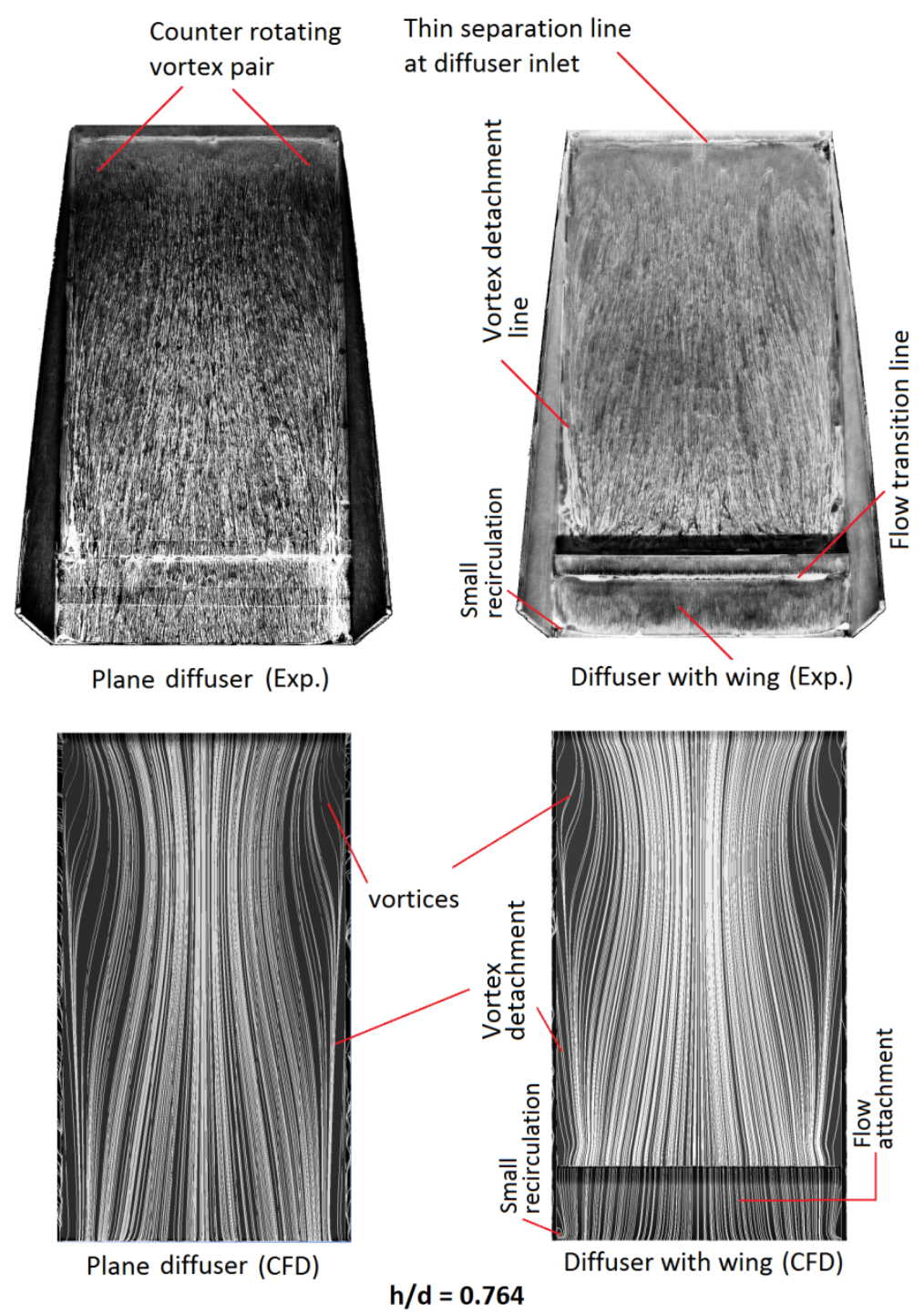

Figure 13: Diffuser surface flow features for the Type A flow regime (force-enhancement) at $h / d=0.764$ (Flow direction from top to bottom) 


\section{Thin separation line \\ at diffuser inlet}

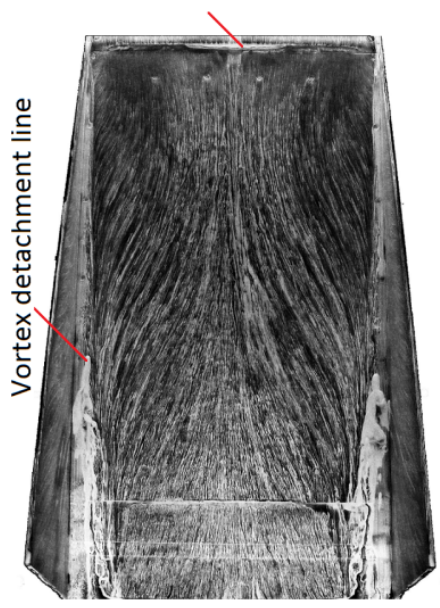

Plane diffuser (Exp.)

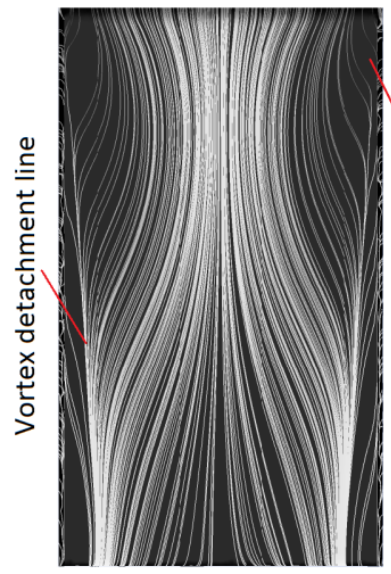

Plane diffuser (CFD)

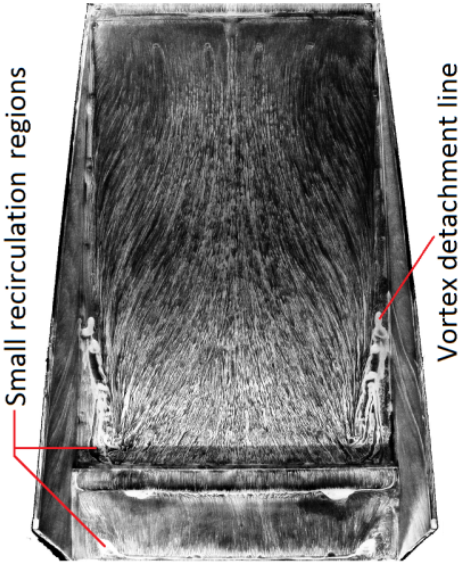

Diffuser with wing (Exp.)

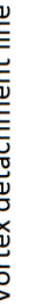

(1)

.

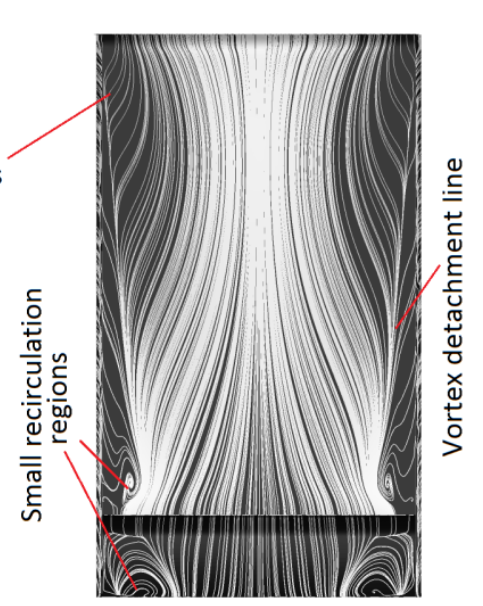

Diffuser with wing (CFD)

$h / d=0.382$

Figure 14: Diffuser surface flow features for the Type A flow regime (force-enhancement) at $h / d=0.382$ (Flow direction from top to bottom)

In the force-enhancement flow regime presented in Figures 13 and 14, the curved pathlines indicate the presence of streamwise vortices along the longitudinal ends of the diffusers. These counter-rotating vortices were also present in the investigations described in [6-9]. The vortices appear to originate from the sides of the diffuser inlet and are propagated in the streamwise direction by the pressure difference inside and outside the diffuser. Moreover, the thin line at the center of the diffuser inlet indicated a separation of the diffuser inlet flow at that location.

The symmetric flow, with the longitudinal vortices on either side of the diffuser centerline $(z / d=0)$, is responsible for the lower pressures along the sides of the diffuser and the symmetrical curvature of the spanwise surface pressure distribution. The curved pathlines of the vortices appear to travel streamwise towards the center of the diffuser. This indicates detachment of the vortex pair from the end plates of the diffuser as the flow traveled 
SAE Journal of Passenger Cars - Mechanical Systems

towards the diffuser exit. However, the dispersal of the curved pathlines towards the diffuser exit indicated that the strength of the vortex pair weakened and detached from the diffuser ramp surface.

On the suction surface of the inverted wing, the thin line that stretched along the span and close to the leading edge of the wing indicates the location of a laminar to turbulent transition. The straightened pathlines downstream of the transition line appeared to be attached to the wing's suction surface. However, there is a recirculation area on the suction surface on either side of the wing, close to the diffuser end plates. Despite this, it is the attachment of the flow to the suction surface of the wing that further lowers the surface pressures at this location and enhances the downforce produced. It is worth noting that the flow features are more pronounced in the case of $h / d=$ 0.382 than at $h / d=0.764$ because a decrease in ride height within the force enhancement flow regime induces an increase in vortex strength.

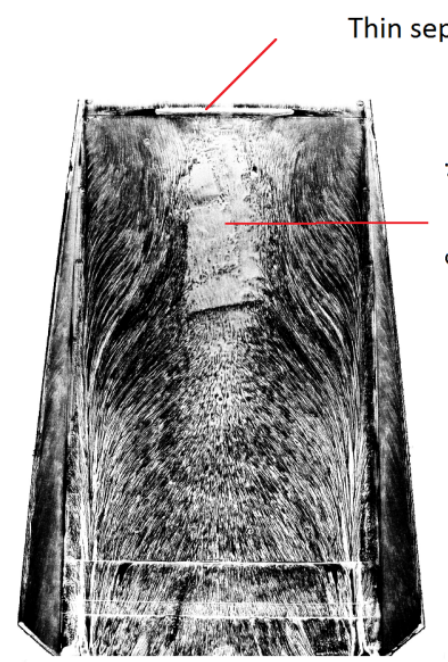

Plane diffuser (Exp.)

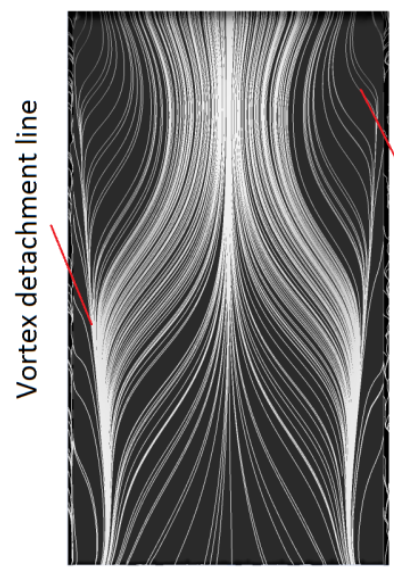

Plane diffuser (CFD)

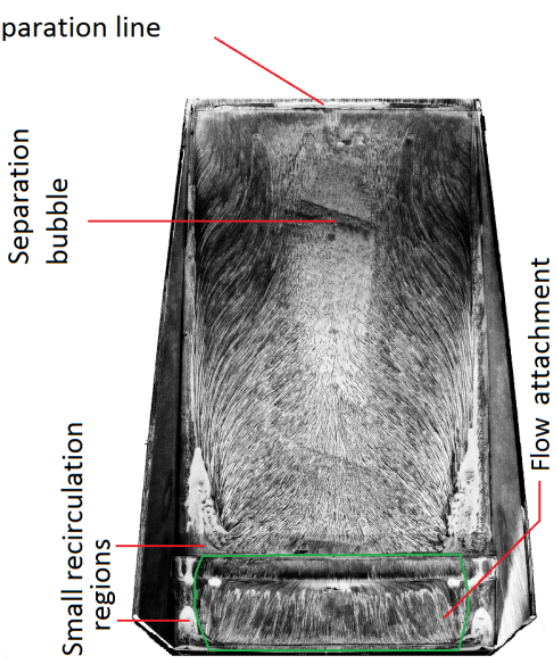

Diffuser with wing (Exp.)

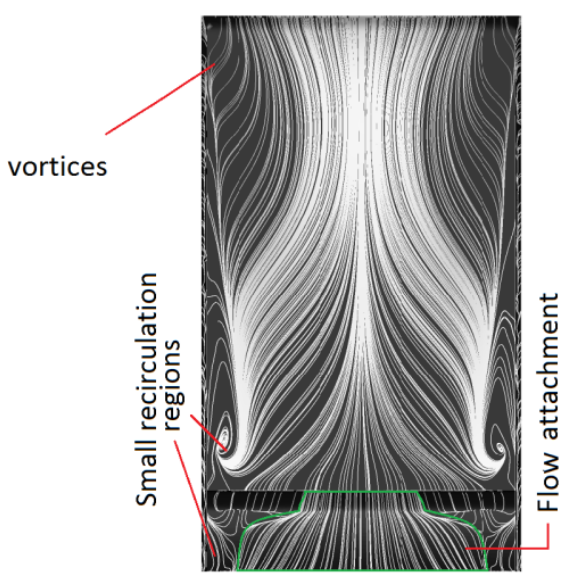

Diffuser with wing (CFD)

$h / d=0.191$

Figure 15: Diffuser surface flow features for the Type B flow regime (maximum-force) at $h / d=0.191$ (Flow direction from top to bottom) 
SAE Journal of Passenger Cars - Mechanical Systems

In Figure 15 the stronger cross flow angle of the curved pathlines in the force-enhancement flow regime, induced by the rotation of the vortices, implies that the strength of the vortex pair has increased. Also, the diffuser flow remains symmetric across the diffuser ramp centerline $(z / d=0)$ and the enhanced vortex strength is responsible for the lower surface pressures close to the endplates relative to those of $h / d=0.382$. The separation line along both lengthwise sides of the diffuser appears to have extended downstream. Also, the thin central separation line along the diffuser inlet has thickened with the separation bubble extending downstream and along the center of the diffuser ramp. These flow features imply that there is an increase in both the streamwise flow velocity into the diffuser and the adverse pressure gradient encountered by the diffuser flow. In addition, the reduced presence of the curved pathlines towards the exit of the diffuser implies that the vortex pair has detached from the ramp. In contrast, the diminished presence of the separation bubble towards the exit of the diffuser infers that the diffuser flow between the vortex pair has reattached to the diffuser ramp.

The thin line across the span of the suction surface of the wing indicates that the laminar-to-turbulent transition remains present. However, the recirculation downstream of the transition line on each lengthwise side of the wing appears to be more enhanced than at $h / d=0.382$, indicating increased flow velocity. Despite that, the presence of the straightened streamlines between both recirculation regions indicates the flow is attached within that area. This is corroborated by the lower surface pressures measured at $x / d=6.29$ for the modified diffuser relative to those of the plane diffuser at the same position.

The flow asymmetry revealed by the surface pressure distribution at the force-reduction ride height of $h / d=0.153$ is confirmed by the surface flow features in Figure 16. The disappearance of one of the longitudinal vortices is responsible for the reduction in downforce produced by this flow regime. A vortex breakdown can be said to have occurred at the location where the vortex was previously present. It also appears that the breakdown of the vortex has extended as a diagonal flow towards the location of the existing vortex. However, the flow reversal of parts of the diagonal flow towards the diffuser inlet and to a larger extent towards the origin of the vortex breakdown indicates that the flow has separated from the ramp in those areas.

Also, the thin separation line found along the span of the diffuser inlet appears to have shifted to the location of the surviving vortex. In addition, the flow detachment of the surviving vortex from the endplate appears to have further extended upstream. Both occurrences indicate that the surviving vortex has been weakened by the increasing adverse pressure gradient encountered by the boundary layer flow entering the diffuser. It is noteworthy to point out that at the force-reduction ride heights, the volume of the underbody flow dominated by the lower surface boundary layer thickness increases. As a result, the slow-moving boundary layer becomes more dominant and is probably responsible for the vortex breakdown, flow reversal and separation as observed on the diffuser ramp surface. 


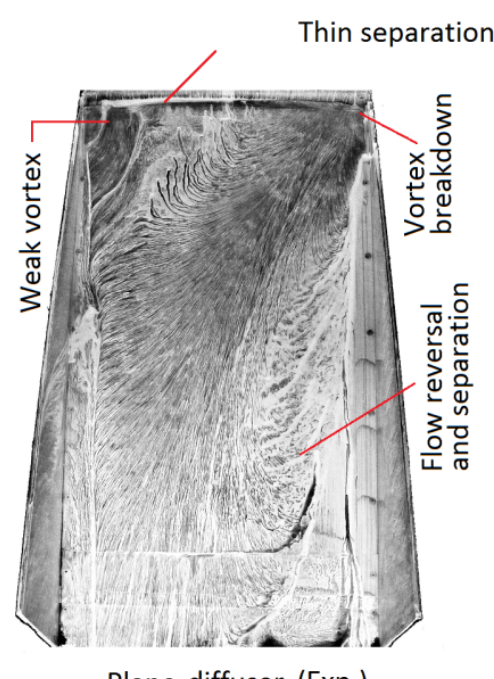

Plane diffuser (Exp.)
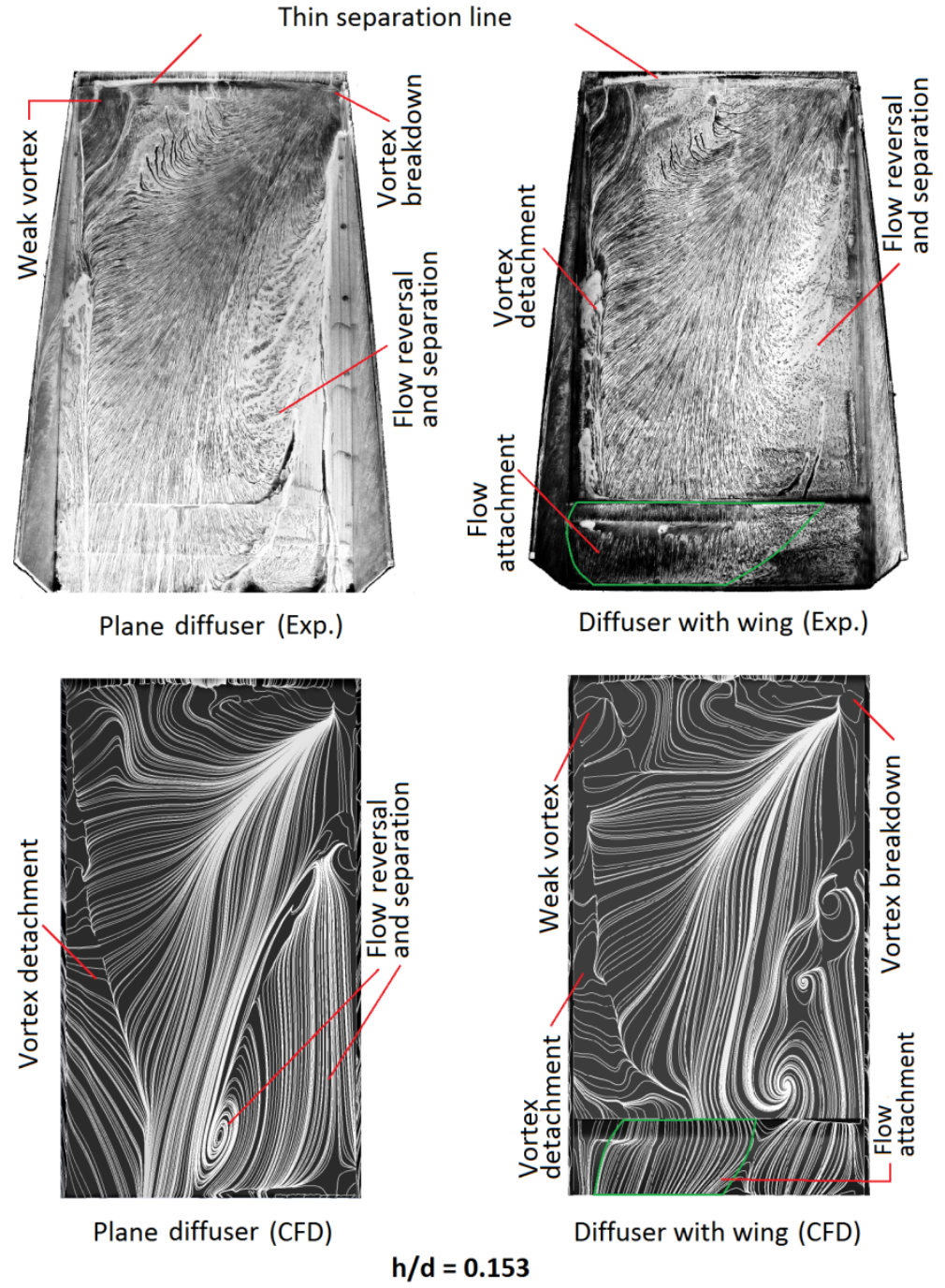

Figure 16: Diffuser surface flow features for the Type $C$ flow regime (force-reduction) at $h / d=0.153$ (Flow direction from top to bottom)

On the suction surface of the wing, the transition line is only present along the half of the wing span, on the side where the surviving vortex exists. The other half of the wing span is largely dominated by reversed flow indicating that the diffuser flow has separated from the suction surface of the wing. Moreover, on the half of the suction surface of the wing where the flow is attached, surface pressures measured along $x / d=6.29$ were lower than those at the same position on the plane diffuser. This indicates that the wing in the modified diffuser induces more suction than the ramp surface of the plane diffuser.

In Figure 17, the surface flow features presented indicate that the diffuser flow regime of the low-force ride height of $h / d=0.064$ is an enhancement of the flow characteristics of the force-reduction flow regime. This is because the low-force flow regime appears largely to comprise flow separation and recirculation. The flow remains asymmetric with the core of the flow recirculation extending diagonally from the location of the nonexisting vortex to the center of the diffuser ramp. 
SAE Journal of Passenger Cars - Mechanical Systems

The separation along the diffuser inlet appears to have extended across three-quarters of the diffuser width. In addition, the flow detachment line along the side where the surviving vortex exists originates from the diffuser inlet. These features show that the flow entering the diffuser is completely dominated by the boundary layer, which encounters a large adverse pressure gradient. Moreover, the transition line on half of the wing's surface (the half where the surviving vortex exists) is barely visible. This implies that the attachment of the flow on the suction surface of the wing is very limited. As illustrated by the spanwise surface pressure distribution along $x / d=6.29$, the decrease in the surface pressures on the wing's surface relative to the surface pressures at the same location of the plane diffuser is minimal. In practice, the downforce (in terms of $C_{L}$ ) produced by the modified diffuser is $1.9 \%$ more than that of the plane diffuser.

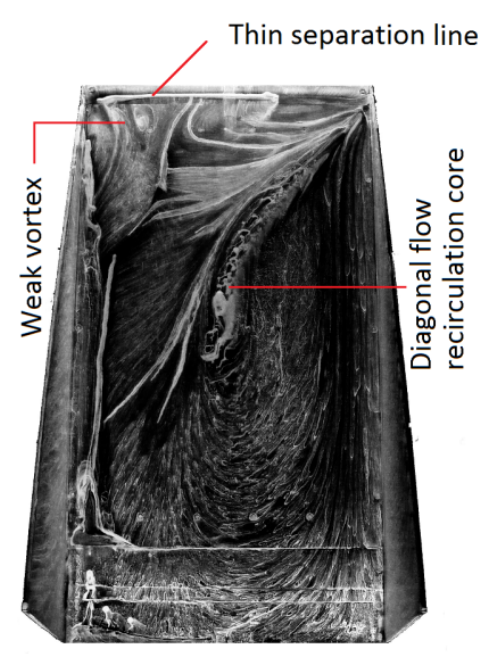

Plane diffuser (Exp.)

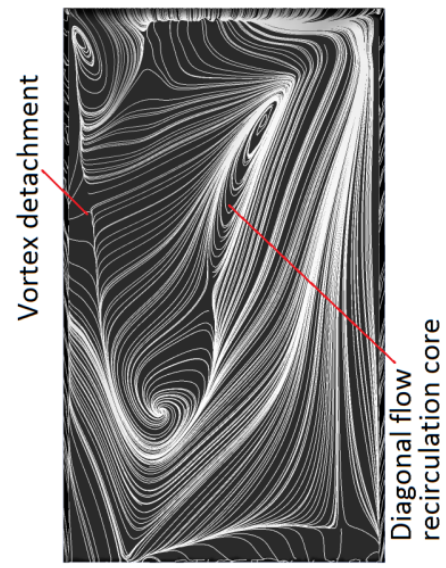

Plane diffuser (CFD)

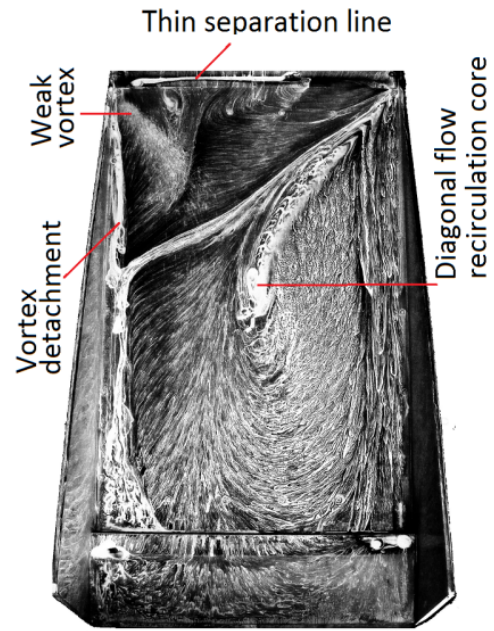

Diffuser with wing (Exp.)

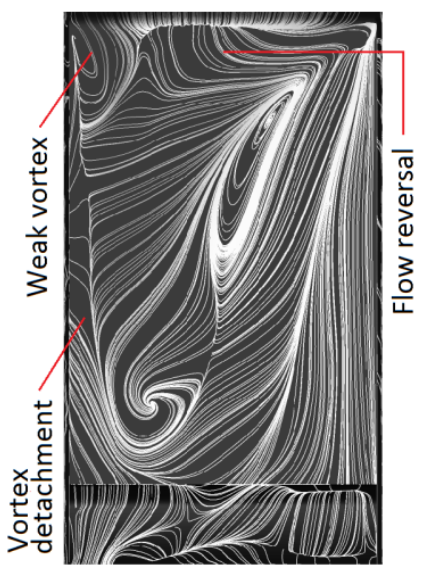

Diffuser with wing (CFD) $h / d=0.064$

Figure 17: Diffuser surface flow features for the Type D flow regime (low-force) at $h / d=0.064$ (Flow direction from top to bottom) 
SAE Journal of Passenger Cars - Mechanical Systems

\section{D. Diffuser Inlet Boundary Layer}

The boundary layer velocity profiles $\left(U / U_{\infty}\right)$ were measured on each side of the diffuser centerline $(z / d=$ 0.363 and -0.363$)$ at the diffuser inlet $(x / d=3.14)$ using laser Doppler velocimetry [20]. The locations on each side of the centerline are approximately outside the core region of the counter-rotating longitudinal vortices of the diffuser and correspond to distances $100 \mathrm{~mm}$ from the spanwise sides of the diffuser. Figure 18 shows that even though the wind tunnel floor boundary layer is removed upstream of the moving ground, the acceleration of the underbody flow produces a boundary layer on the moving ground plane, as well as on the diffuser inlet surface.

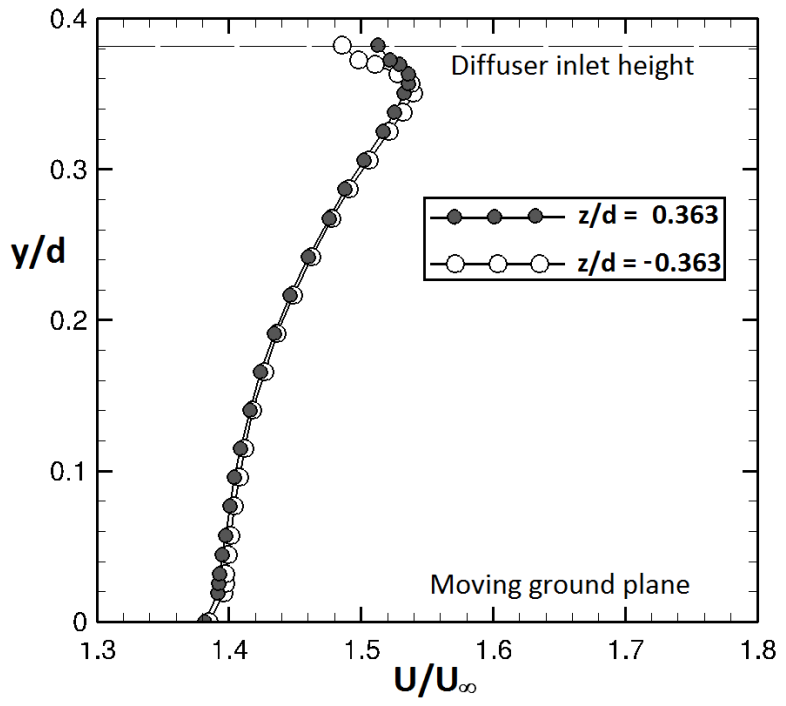

(a)

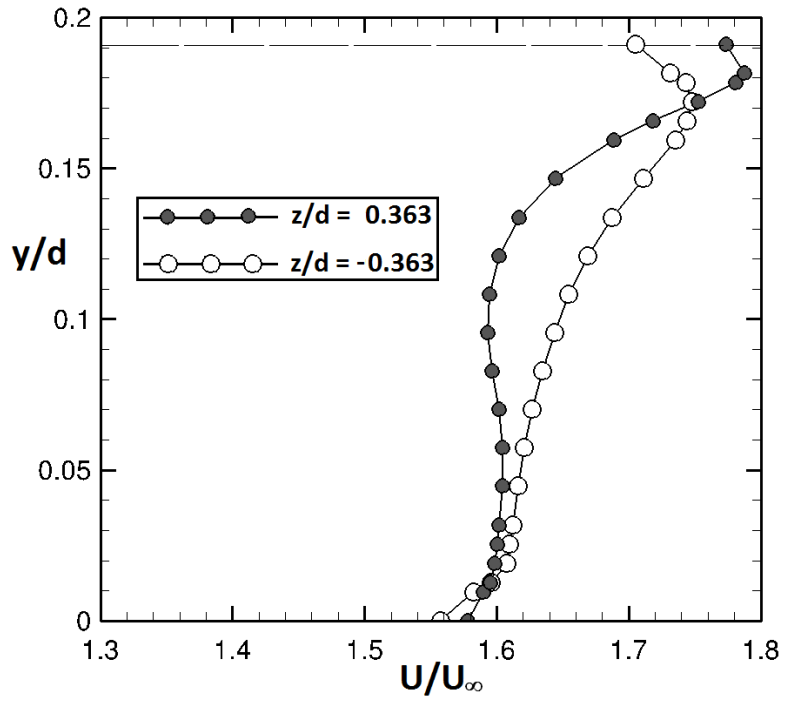

(b)

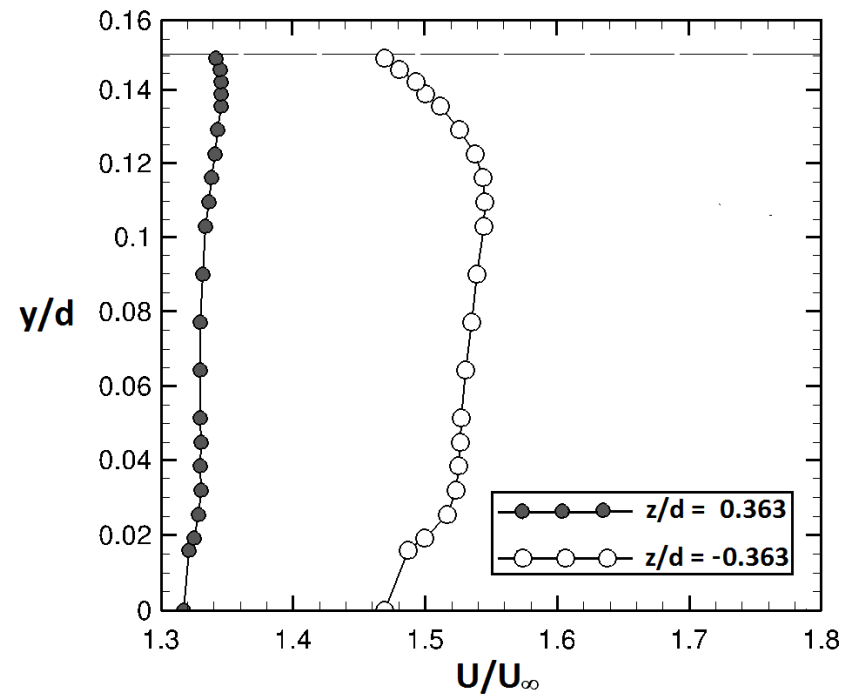

(c)

Figure 18: Boundary layer velocity profiles measured at each side $(\boldsymbol{z} / \boldsymbol{d}=\mathbf{0 . 3 6 3}$ and $-\mathbf{0 . 3 6 3})$ of diffuser inlet center point $(\boldsymbol{x} / \boldsymbol{d}=\mathbf{3 . 1 4})$ for: (a) $h / d=0.382$ (b) $h / d=0.191$ (c) $h / d=0.153$

The velocity profiles for the force-enhancement (type A) flow regime shown in Figure 18a indicates that the peak flow velocities on both sides of the diffuser inlet centerline are reasonably similar. This further corroborates 
SAE Journal of Passenger Cars - Mechanical Systems

the symmetry associated with the force-enhancement flow regime. In the maximum-force (type B) regime (Figure 18b), the boundary layer profiles indicate flow asymmetry at the diffuser inlet, highlighted by a region $(y / d=0.031$ to 0.165$)$ of velocity variation between the two profiles $(1.4 \%$ lower between highest velocities in the region). However, the surface pressure distribution (Figure 12d) and on-surface flow visualisation (Figure 15) for the maximum-force regime indicate the reasonable existence of flow symmetry within the diffuser. Moreover, as in the maximum-force case where boundary layer velocities are lower at $z / d=0.363$ compared to those at $z / d=-0.363$, a similar occurrence is present in force-reduction (type C) regime (Figure 18c). However, in the type $\mathrm{C}$ case the boundary layer velocities on the side of the diffuser where vortex breakdown occurred (at $z / d=0.363$ ) are significantly lower than the velocities at the other side (at $z / d=-0.363$ ) where there is a surviving vortex (13\% lower between measured peak velocities). This implies that the onset of vortex breakdown responsible for downforce loss in the force reduction and low downforce ride heights begins to occur at the maximum downforce ride height.

Despite these occurrences, the velocity profiles of the maximum-force and force-reduction regimes suggest the presence of a region of relatively constant velocity within the distance between the boundary layers of the diffuser inlet and the ground plane. This occurrence indicates that although the reduction of ride height beyond that of maximum downforce enhances flow separation at the diffuser inlet, a complete merging of the boundary layers does not occur. Hence, the merging of the boundary layers is not entirely responsible for the flow asymmetry associated with the force-reduction and low-downforce ride heights. However, the velocity of the boundary layer reduces with decreasing ride height. Furthermore, the slower boundary layer flow velocities on the side of the diffuser inlet where vortex breakdown occurs appear to have induced the vortex breakdown on that side.

\section{E. Skin Friction Drag}

As shown in Figure 19, skin friction behavior along the ramp surface of the diffuser was ascertained using CFD at the maximum-downforce regime ride height $(h / d=0.191)$. CFD predictions were computed along the center of the diffuser ramp length $(z / d=0)$ and along each side of the central length where the counter-rotating vortices are approximately located $(z / d=0.490$ and -0.490$)$. For both the plane and modified diffusers, the initial observation is that there is a reasonable symmetry in skin friction coefficient $\left(C_{f}\right)$ distribution along $z / d=$ 0.490 and -0.490 (Figure 19) as a result of the diffuser flow symmetry associated with the vortex pair. For the plane diffuser, the substantial drop in $C_{f}$ around the start of the diffuser ( $x / d=3.14$ to 3.5$)$ is due to the peak velocity at the diffuser inlet gradually decelerating thus inducing localized boundary layer growth. Downstream of the diffuser inlet $\left(x / d=3.5\right.$ to 5.6) along $z / d=0.490$ and -0.490 (Figure 19a and 19c), $C_{f}$ increases and 
SAE Journal of Passenger Cars - Mechanical Systems

then decreases due to the vortex strength being strong at the early part of the diffuser and gradually weakening as it travels downstream towards the diffuser exit. At the exit, the sudden short rise in $C_{f}$ is as a result of the turbulent wake at the near-wake region of diffuser bluff body.

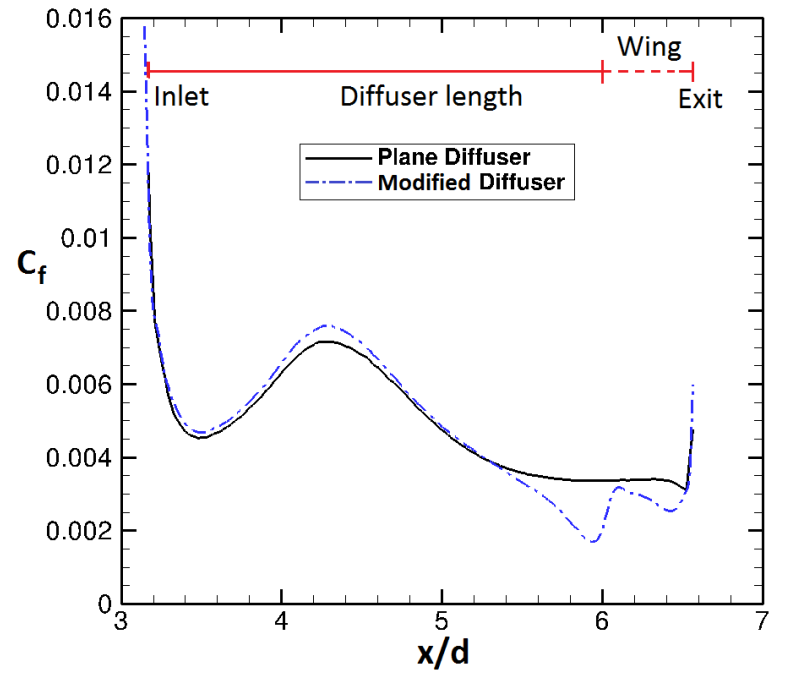

(a)

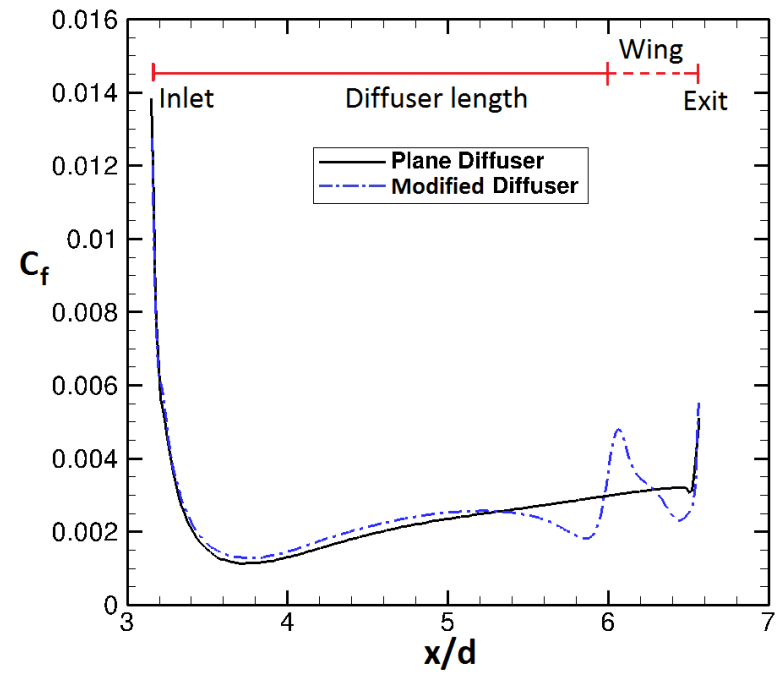

(b)

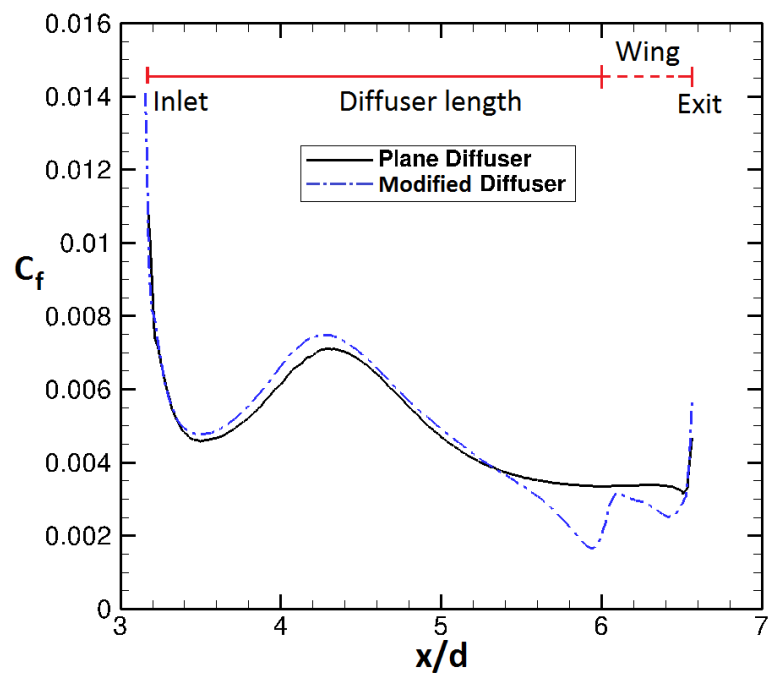

(c)

Figure 19: CFD predictions of skin friction coefficient $\left(C_{f}\right)$ at maximum downforce ride height $(h / d=0.191)$ for the plane and modified diffusers on: (a) $z / d=0.490$, (b) $z / d=0$, and (c) $z / d=-0.490$

In the case of the plane diffuser centerline $C_{f}$ distribution at (Figure 19b), there is a gradual rise in $C_{f}$ between the significant drop in $C_{f}$ around the diffuser inlet region and a small sharp rise in $C_{f}$ at the diffuser exit. This is because there is no presence of the longitudinal vortices along the diffuser centerline and at the diffuser exit there is a turbulent near-wake. The gradual increase towards the diffuser exit of $C_{f}$ on the diffuser ramp centerline is, as detailed in [20], due to the turbulent boundary layer profile being less full at the early part of the diffuser and more full towards the diffuser exit. Thus, as the boundary layer gradually becomes more turbulent, $C_{f}$ gradually increases. 
SAE Journal of Passenger Cars - Mechanical Systems

The addition of the inverted wing in the case of the modified diffuser induces a change in the distribution of $C_{f}$ on the diffuser ramp surface from $x / d=5.30$ to 6.54 (Figure 19). Between $x / d=5.3$ to 6.0, there is a drop in the local skin friction $\left(C_{f_{x}}\right)$ as a result of the localized flow deceleration induced by the leading edge of the wing. Within the region $x / d=6.0$ to 6.54 , which encompasses the length of the wing, there is a rise and drop in skin friction. The rise in $C_{f}$ is as a result of the viscous forces acting on the flow as it negotiates and accelerates over the early part of the wing. However, downstream of the early part of the wing, there is a drop in $C_{f}$ as the flow decelerates towards the diffuser exit. Table 1 indicates that between the two diffusers, there is no difference in the streamwise-length-averaged skin friction $\overline{\bar{C}}_{f}$ as calculated using Eq. (3) along $z / d=$ $0.490,0$, and -0.490 .

Where:

$$
\overline{\bar{C}}_{f}=\frac{1}{l_{d}} \int_{x / d=3.14}^{x / d=6.57} C_{f} d x
$$

and $l_{d}$ is the diffuser ramp length $=0.563 \mathrm{~m}$

Table 1: Streamwise-length-averaged skin friction along $z / d=0.490,0$, and -0.490 for the plane and modified diffusers at $h / d=0.191$

\begin{tabular}{cccc}
\hline \multicolumn{1}{c}{$\boldsymbol{h} / \boldsymbol{d}$} & $\begin{array}{c}\overline{\overline{\boldsymbol{C}}}_{\boldsymbol{f}} \text { at } \\
(\mathbf{z} / \boldsymbol{d}=\mathbf{0 . 4 9 0})\end{array}$ & $\begin{array}{c}\overline{\overline{\boldsymbol{C}}}_{\boldsymbol{f}} \text { at } \\
(\mathbf{z} / \boldsymbol{d}=\mathbf{0})\end{array}$ & $\begin{array}{c}\overline{\overline{\boldsymbol{C}}}_{\boldsymbol{f}} \text { at } \\
(\boldsymbol{z} / \boldsymbol{d}=-\mathbf{0 . 4 9 0})\end{array}$ \\
\hline 0.191 (Plane Diffuser) & 0.0046 & 0.0023 & 0.0046 \\
0.191 (Modified Diffuser) & 0.0046 & 0.0023 & 0.0046 \\
\hline
\end{tabular}

\section{Discussion}

The investigations in this study, aimed at understanding the physics of the ground-effect diffuser flow, have supported the findings from previous studies $[8,9]$ on this subject. As observed, there are four distinct flow regimes determined over the wide range of ride heights tested. At high ride heights, the force-enhancement flow regime dominates the diffuser flow. Subsequent reductions in ride height enhanced the flow features of the forceenhancement flow regime as seen in the maximum-force flow regime. In the force-reduction flow regime, a transformation of the symmetric flow to an asymmetric one occurred with further reductions in ride height. Additional ride height reductions beyond the force-reduction region further intensified the flow characteristics of the force-reduction flow regime as observed in the low-force flow regime. Of note is the aerodynamic performance influence on the diffuser by the diffuser inlet peak suction, streamwise vortex shedding along the longitudinal ends of the diffuser and the adverse pressure gradient of the diffuser flow.

From the force-enhancement to the maximum-force flow regime, the surface pressures at the diffuser inlet reduced with a decrease of ride height. This indicated that the streamwise velocity of the constrained flow 
SAE Journal of Passenger Cars - Mechanical Systems

underneath the underbody of the test model was also increased by lowering ride height. As stated by Bernoulli's principle, an increase in flow velocity comes with a corresponding decrease in pressure. In the same way, the suction-generating longitudinal vortex pair along each lengthwise side of the diffuser increased in strength as the ride height was decreased. However, the decrease in ride height intensified the adverse pressure gradient encountered by the diffuser flow. In the maximum force flow regime, the appearance of a separation bubble that extended downstream of the diffuser inlet was the result of the increasing adverse pressure gradient.

In the force-reduction and low-force flow regimes, the influence of boundary layer flow became more prevalent. This was because the lower ride heights of the force-reduction and low-force flow regimes implied that the diffuser flow entering the diffuser was increasingly boundary layer dominant. The slow-moving boundary layer flow entering the diffuser provoked a breakdown of one of the pair of longitudinal vortices. The flow became increasingly separated from the diffuser ramp with flow reversal and recirculation dominating the diffuser flow. In addition, the decrease in ride height from the force-reduction to the low-force regime reduced the peak suction at the diffuser inlet and the strength of the surviving vortex with also an increase in intensity of the adverse pressure gradient.

In the study of Senior [9], however, an arbitrary switching of the vortex breakdown from one vortex to the other was observed when the ride height was lowered from the force-reduction to low-force regime. Although the force-reduction (type $\mathrm{C}$ regime) flow entering the diffuser is boundary layer dominant, and partly the cause of the vortex breakdown, imperfections of the underbody surface of the test model can also induce a bi-stability of the asymmetric diffuser flow present in the type $\mathrm{C}$ flow regime. However, the surface pressures along the sides of the diffuser, where both longitudinal vortices are located, show that the suction level on one side (at $x / d=3.63$ ) is about $3.4 \%$ higher than the other. This also switched from side to side between the force-enhancement and maximum-force regimes. In addition, the vortex on the side with the higher suction level appeared to be the solitary vortex in the force-reduction and low-force flow regimes. This behavior likewise occurred in the investigations of Senior [9]. Therefore, it can be asserted that the strength of each of the longitudinal vortices dictates which survives in the force-reduction and low-force flow regimes. The (slightly) stronger vortex with the higher suction not only appeared to have survived in the force-reduction flow regime, but also appeared to have induced a diagonal flow across the diffuser as shown by the diagonal flow streamlines.

The lower surface pressures on the suction surface of the wing relative to those of the plane diffuser ramp surface at the same location indicated that the wing was solely responsible for the increase in downforce. This was due to the pressure gradient induced by the flow curvature underneath the suction surface of the wing and this also influenced the attachment of the flow to the wing's suction surface. As a consequence, the flow velocity across the wing's surface increased, thereby causing a decrease in static pressure as dictated by Bernoulli's 
SAE Journal of Passenger Cars - Mechanical Systems

principle. Thus, downforce was increased with a second static pressure drop and recovery region induced near the diffuser exit.

With the use of the inverted wing, downforce increased for the representative flow regime ride heights $(h / d=$ $0.764,0.382,0.191,0.153$, and 0.064$)$ by $+12 \%,+4.0 \%,+3.0 \%,+2.4 \%$, and $+1.9 \%$ respectively. Likewise, the use of the wing in the diffuser flow was also accompanied by a corresponding drag increase due to added profile drag of the wing. For the representative ride heights drag increased by $+11 \%,+6.7 \%,+1.9 \%,+2.6 \%$, and $+0.3 \%$. CFD investigations indicated that skin-friction along the diffuser ramp surface rapidly increased as flow accelerates round the wing leading edge but then gradually reduced downstream as the flow decelerates towards the diffuser exit. Despite this, the lengthwise-averages for skin friction predicted on both sides of the modified diffuser ramp centerline are equal to those of the plane diffuser. This appears to indicate that the wing may have slightly increased the peak velocities of vortices at the diffuser inlet.

Preliminary CFD investigations also indicated that an increase in chord length, $c$, of the inverted wing will increase downforce due to a larger suction surface area and thus increased region of induced pressure gradient. However, an increase in $c$ can hinder the aerodynamic performance of the diffuser due to the increased profile drag and blockage of the diffuser flow channel. Also, a second pressure drop near the equivalent pressure drop at the diffuser inlet can be too close to each other to allow an adequate pressure recovery from the initial pressure drop at the inlet before a second pressure drop is induced. Hence, an effective aerodynamic performance is achieved with a wing chord length, $c$ between $15 \%$ and $25 \%$ of the diffuser length [20]. Also, to mitigate flow separation above the inverted wing, good aerodynamic performance is achieved at $\alpha=\theta$ with the gap between the wing and the ramp surface at about $50 \%$ of the diffuser ramp boundary layer thickness.

\section{Concluding Remarks}

The aerodynamic performance characteristic of a diffuser as a downforce-generating device on a generic bluff body has been underlined in this paper. In near proximity to the ground, the diffuser inlet converts highvelocity flow travelling through it to low static pressures and, towards the diffuser exit, the flow gradually becomes a low-velocity flow with a recovery of static pressures at the diffuser exit. Under a wide range of ride heights, the diffuser flow exhibits four distinct flow regimes.

In the force-enhancement flow regime longitudinal counter-rotating vortices are formed, and the adverse pressure gradient encountered by the flow detaches the vortices as they approach the diffuser exit. In the maximum-force flow regime, the downforce produced by the diffuser reaches it maximum due to enhanced suction at the diffuser inlet. Also, the vortices are strengthened and the flow around the center of the diffuser separates before reattaching towards the diffuser exit. The force-reduction flow regime induces a breakdown of 
SAE Journal of Passenger Cars - Mechanical Systems

one of the vortices and causes the 3-D diffuser flow to become asymmetric. In addition, the unsteady diffuser flow becomes increasingly separated from the diffuser ramp, flow reversal occurs, and downforce reduces. The lowforce flow regime is largely dominated by boundary layer flow. Also, the surviving vortex is severely weakened with flow separation and recirculation becoming increasingly prevalent. As a result the flow is very unstable and a low downforce is generated.

The novel application of the suction surface of the inverted wing to enhance flow attachment towards the exit of the diffuser appeared to have also increased downforce. The suction surface of the wing enhances flow attachment by increasing flow velocity induced by the curvature of the wing. However, between the wing and the diffuser ramp, the diffuser flow through the gap generates low pressure but the suction surface generates lower surface pressures. This implies that the net surface pressure entirely induced by the wing remains negative. As a result, a secondary pressure recovery region develops towards the exit of the diffuser. Through this action, average surface pressures in that location become negative and additional downforce is produced by the inverted wing.

The application of the inverted wing was observed to increase downforce to a high of $\sim 12 \%$ at the highest ride height of the type A force-enhancement flow regime and by $\sim 3 \%$ at the maximum downforce ride height (type B). However, the percentage downforce increments gradually reduced with decreasing ride height as a result of the small recirculation regions at either end of the wing suction surface induced by the diffusing longitudinal vortices. Furthermore, the use of the wing as a passive flow control device had no effect on the type C forcereduction flow regime because it was positioned far away from the diffuser inlet and thus, had no control on the vortex breakdown linked to the force-reduction regime. Effectual aerodynamic performance of the wing is influenced by its angle of attack $(\alpha)$ and the gap between its pressure side and the ramp surface.

\section{References}

[1] Zhang, X., Toet, W., and Zerihan, J., "Ground Effect Aerodynamics of Racing Cars" Applied Mechanics Review, Vol. 59 No. 1, pp. 33-49, Jan. 2006.

[2] Toet, W., “Aerodynamics and Aerodynamic Research in Formula 1", The Aeronautical Journal, Vol. No. 117, No. 1187, pp. 1-25, Jan. 2013.

[3] Katz, J., "New Directions in Race Car Aerodynamics", $2^{\text {nd }}$ Revised ed., Bentley Publishers, Cambridge, Massachusetts, Chap. 6. pp. 221-222, Mar. 2006.

[4] Cooper, K. R., Bertenyi, T., Dutil, G., Syms, J., and Sovran, G., "The Aerodynamic Performance of Automotive Underbody Diffusers,” SAE Technical Paper 980030, Feb. 1998.

[5] Cooper, K. R., Sovran, G., and Syms, J., "Selecting Automotive Diffusers to Maximise Underbody Downforce," SAE Technical Paper 2000-01-0354, Mar. 2000.

[6] George, A. R., “Aerodynamic Effects of Shape, Camber, Pitch on Ground-vehicle Bodies”, ASME Journal of Fluids Engineering, Vol. 103, No. 4, pp. 631-637, Dec. 1981. 
[7] George, A. R. and Donis, J., "Flow Patterns, Pressures and Forces in the Underside of Idealised Ground Effect Vehicles", Proceedings of the ASME Fluids Engineering Division Symposium on Aerodynamics of Transportation II, Vol. 7, pp. 69-79, Dec. 1983.

[8] Senior, A. E., and Zhang, X., "An Experimental Study of a Diffuser in Ground Effect", 38 ${ }^{\text {th }}$ AIAA Aerospace Sciences Meeting and Exhibit, Paper No. 2000-0118, Reno, Nevada, USA, Jan. 2000.

[9] Senior, A., “The Aerodynamics of a Diffuser Equipped Bluff Body in Ground Effect," Ph.D. Thesis, University of Southampton, UK, April 2002.

[10] Jowsey, L., “An Experimental Study of Automotive Underbody Diffusers", Ph.D. Thesis, Loughborough University, UK, 2013.

[11] Puglisevich, L. S., "Large Eddy Simulation for Automotive Vortical Flows in Ground Effect", Ph.D. Thesis, Loughborough University, UK. 2013.

[12] Marklund, J., "Under-body and Diffuser Flows of Passenger Vehicles", Ph.D. Thesis, Chalmers University of Technology, Sweden, 2013.

[13] Ehirim, O. H., Knowles, K., Saddington, A. J., and Finnis, M. V., "Ground-effect Aerodynamics: an Analytical Review on Ground-effect Diffusers”, ASME Journal of Fluids Engineering, 2018.

[14] Gibson, M. M., Verriopoulos, C. A., Vlachos, N. S., "Turbulent Boundary Layer on a Mildly Curved Convex Surface Part 1: Mean Flow and Turbulence Measurements," Experiments in Fluids, Vol. 2, No. 1, pp. 17-24, Mar. 1984.

[15] Muck, K. C., Hoffmann, P. H. and Bradshaw, P., "The Effect of Convex Surface Curvature on Turbulent Boundary Layers," Journal of Fluid Mechanics, Vol. 161, pp. 347-369, Dec. 1985.

[16] So, M. C. R. and Mellor, G. L., "Experiment on Convex Curvature Effects in Turbulent Boundary Layers," Journal of Fluid Mechanics, Vol. 60, No. 1, pp. 43-62, Aug. 1973.

[17] Bradshaw, P., “Effects of Streamline Curvature on Turbulent Flow,” AGARDograph AG-169, Jan. 1973.

[18] Knowles, K. and Finnis, M. V., "Development of a New Open-Jet Wind Tunnel and Rolling Road Facility" In: 2nd MIRA International Conference on Vehicle Aerodynamics, Coventry, UK, Oct. 1998.

[19] Zerihan, J., and Zhang, X., “Aerodynamics of a Single-Element Wing in Ground Effect,” Journal of Aircraft, Vol. 37, No. 6, pp. 1058-1064, Dec. 2000.

[20] Ehirim, O. H., “Aerodynamics and Performance Enhancement of a Ground-effect Diffuser”, Ph.D. Thesis, Cranfield University, Shrivenham, UK. 2017. http://dspace.lib.cranfield.ac.uk/handle/1826/13211

[21] Zhang, X., Zerihan, J., Ruhrmann, A., and Deviese, M., "Tip Vortices Generated by a Wing in Ground Effect," 11th International Symposium on Applications of Laser Techniques to Fluid Mechanics, Instituto Superior Tecnico, Lisbon, Portugal, 2002.

[22] Zhang, X., and Zerihan, J., "Aerodynamics of a Double Element Wing in Ground Effect," AIAA Journal, Vol. 41, No. 6, pp. 1007-1016, Jun. 2003.

[23] Zhang, X., and Zerihan, J., "Edge Vortices of a Double-Element Wing in Ground Effect," Journal of Aircraft, Vol. 41, No. 5, pp. 1127-1137, Oct. 2003.

[24] Abernethy, R. B., Benedict, R. P., and Dowdell, R. B., “ASME Measurement Uncertainty," ASME Journal of Fluids Engineering, Vol. 107, No. 2, pp. 161-164, Jun. 1985.

[25] Moffat, R. J., "Describing the Uncertainties in Experimental Results," Experimental Thermal and Fluid Science. Vol. 1, No. 1, pp 3-17, Jan. 1988. 
SAE Journal of Passenger Cars - Mechanical Systems

[26] Maskell, E. C., "A Theory of the Blockage Effects of Bluff Bodies and Stalled Wings in a Closed Wind Tunnel”, ARC R\&M 3400, 1965.

[27] Barlow, J. B., Rae, W. H. and Pope, A., “Low-Speed Wind Tunnel Testing”, $3^{\text {rd }}$ Edition, Wiley \& Sons, New York, 1999.

[28] ANSYS ICEM CFD, Release (17.2), 2600 ANSYS Drive, Canonsburg, PA 15317, USA. www.ansys.com, 2016.

[29] ANSYS FLUENT, Release (17.2), 2600 ANSYS Drive, Canonsburg, PA 15317, USA. www.ansys.com, 2016.

[30] Menter, F. R., "Two-Equation Eddy-Viscosity Turbulence Models for Engineering Applications", AIAA Journal, Vol. 32, No. 8, pp. 1598-1605, Aug. 1994.

[31] Menter, F.R., Langtry, R., and Völker, S., “Transition Modelling for General Purpose CFD Codes.” Flow, Turbulence and Combustion, Vol. 77, pp. 277-303, August, 2006.

[32] Langtry, R. B., and Menter, F. R., "Correlation-Based Transition Modeling for Unstructured Parallelized Computational Fluid Dynamics Codes,” AIAA Journal, Vol 47, No. 12, pp. 2894-2906, December, 2009.

[33] Walters, D. K. and Cokljat, D., “A Three-Equation Eddy-Viscosity Model for Reynolds-Averaged Navier-Stokes Simulations of Transitional Flow," ASME Journal of Fluids Engineering, Vol. 130, No. 12, 121401, October, 2008.

[34] Spalart, P. R., Jou, W-H., Strelets, M., Allmaras, S. R., "Comments on the Feasibility of LES for Wings and on a Hybrid RANS/LES Approach," Proceedings of 1st AFOSR International Conference on DNS/LES. Louisiana Tech University, pp. 137-147, Aug. 1997.

[35] Spalart, P., "Strategies for Turbulence Modelling and Simulations," International Journal of Heat and Fluid Flow, Vol. 21, No. 3, 252-263, Jun. 2000.

[36] Spalart, P. R., Deck, S., Shur, M. L., Squires, K. D., Strelets, M. K., \& Travin, A., “A New Version of Detachededdy Simulation, Resistant to Ambiguous Grid Densities," Theoretical and Computational Fluid Dynamics, Vol. 20, No. 3, 181-195, May 2006.

[37] Shur, M. L., Spalart, P. R., Strelets, M. K., \& Travin, A. K., “A Hybrid RANS-LES Approach with Delayed-DES and Wall-modelled LES Capabilities," International Journal of Heat and Fluid Flow, Vol. 29, No. 6, pp. 16381649, Dec. 2008.

[38] Spalart, P. R., “Detached-Eddy Simulation,” Annual Review of Fluid Mechanics, Vol. 41, No. 1, pp. 181-202, Jan. 2009.

[39] Spalart, P. R., "Young-Person's Guide to Detached-Eddy Simulation Grids," NASA Langley Technical Report, NASA/CR-2001-211032, July 2001.

[40] Ehirim, O. H., Knowles, K., Saddington, A. J., and Finnis, M. V., “Aerodynamics of a Convex Bump on a Groundeffect Diffuser”, ASME Journal of Fluids Engineering, Vol. 140, No. 9, pp. 091102-091102-11 2018. 